\title{
A Review of Predictive Nonlinear Theories for Multiscale Modeling of Heterogeneous Materials
}

\author{
Karel Matouša,*, Marc G.D. Geers ${ }^{\mathrm{b}}$, Varvara G. Kouznetsova ${ }^{\mathrm{b}}$, Andrew Gillman ${ }^{\mathrm{a}}$ \\ ${ }^{a}$ Department of Aerospace and Mechanical Engineering, University of Notre Dame, Notre Dame, IN, 46556, USA. \\ ${ }^{b}$ Department of Mechanical Engineering, Eindhoven University of Technology, Eindhoven, The Netherlands.
}

\begin{abstract}
Since the beginning of the industrial age, material performance and design have been in the midst of innovation of many disruptive technologies. Today's electronics, space, medical, transportation, and other industries are enriched by development, design and deployment of composite, heterogeneous and multifunctional materials. As a result, materials innovation is now considerably outpaced by other aspects from component design to product cycle. In this article, we review predictive nonlinear theories for multiscale modeling of heterogeneous materials. Deeper attention is given to multiscale modeling in space and to computational homogenization in addressing challenging materials science questions. Moreover, we discuss a state-of-the-art platform in predictive image-based, multiscale modeling with co-designed simulations and experiments that executes on the world's largest supercomputers. Such a modeling framework consists of experimental tools, computational methods, and digital data strategies. Once fully completed, this collaborative and interdisciplinary framework can be the basis of Virtual Materials Testing standards and aids in the development of new material formulations. Moreover, it will decrease the time to market of innovative products.
\end{abstract}

Keywords: Predictive Science, Image-Based Multiscale Modeling, Computational Homogenization, High Performance Computing, Co-designed Simulations and Experiments, Verification and Validation

\section{Need for Predictive Multiscale Computational Science and Engineering in Materials Design}

Over the past decade, an abundance of new processing conditions from the material science community has ushered development of materials with unique properties. Similarly, design of machine parts pushes material science towards discoveries of materials that are lighter, stronger, thermally and electromagnetically shielded, multifunctional, etc., and the processing conditions that will create them. This constant push and pull between material science, physics/mechanics, and materials design creates the need for predictive modeling tools that accurately describe the physical phenomena at each length and time scale with the resolution required in product design and safety assessment (see Figure 1(a)).

For a long time, development of new materials has been guided by experience and empirical correlations between processing conditions and desired performance [1]. Recently, this ad hoc approach is being challenged by the Integrated Computational Materials Engineering (ICME) paradigm [2-5]. ICME combines both bottom-up and top-down modeling and simulation strategies and aims to reduce the time to market of innovative products. It is concerned with multiple spatial and temporal scales of structural hierarchy typical of materials with microstructure. Moreover, it synthesizes information and data from both experiments and simulations across time scales and/or length scales. A report by the National Research Council of the National Academies [3] describes ICME as a transformational discipline for improved competitiveness and national security. It also views it as a grand challenge: "The grand challenge for the field of materials science and engineering is to build an ICME capability for all classes and applications of materials."

*Corresponding author, kmatous@nd.edu

Preprint submitted to Elsevier

September 26, 2016

(C) 2016. This manuscript version is made available under the Elsevier user license http://www.elsevier.com/open-access/userlicense/1.0/ 


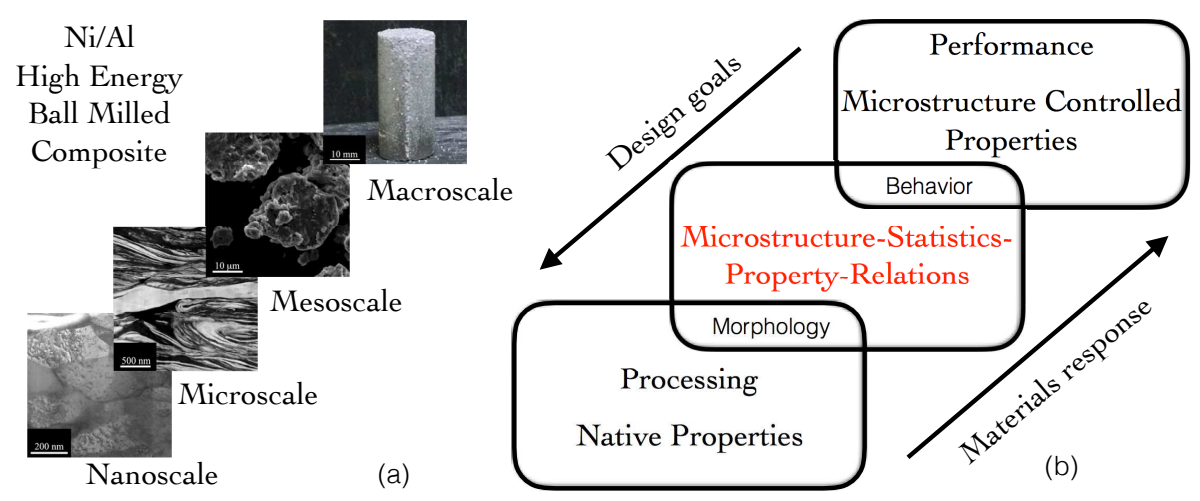

Figure 1: (a) Hierarchy of material scales from Nano- to Macroscale for Ni/Al composite produced by high energy ball milling at the Center for Shock Wave-processing of Advanced Reactive Materials (C-SWARM) at the University of Notre Dame. (b) Microstructure-Statistics-Property Relations.

To address this grand challenge, no single discipline can be called upon exclusively, and knowledge from several branches of science and engineering are needed. In particular, collaborations across mechanics, physics, chemistry, materials science, applied mathematics, data science, and scientific computing are required. Experimental investigations should keep in mind computational needs and vise versa. Abstraction of mathematical tools (i.e. mathematical complexity) should be accessible for easier engineering applicability. Scientific computing should produce libraries that are easy to use and open doors to wider utilization of high performance computing. This is not a trivial task and constitutes the real challenge for the traditional materials science discipline. Thus, a cultural change in the engineering and science fields is needed, and education of a new workforce with diverse skills is paramount for future success.

Methods and tools that form the basis of ICME are essentially those that constitute and expand the elements of "Materials Innovation Infrastructure" (MII) in the Materials Genome Initiative [6]:

- Experimental Tools

- Testing and full-field in situ measurements with co-designed simulations

- Material characterization and microstructure representation

- Unit manufacturing processes - casting, cutting, surface treatment, machining, etc.

- Sensors and control systems

- Computational Tools

- Single scale - ab initio, molecular/atomistics and continuum - models

- Hierarchical, concurrent multiscale models and model reduction

- Verification, validation and uncertainty quantification

- High performance computing and software engineering

- Digital Data - whether derived from computations or experiments

- Data science and material informatics

- Machine learning and computational statistics

- Decision-based system design and optimization

- Material databases 
These integrated computational, experimental, and data informatics tools should span the entire materials landscape, improve predictive capabilities, and execute on the next-generation of supercomputers. Such an infrastructure will enable rapid and holistic engineering design that seamlessly integrates into existing product design frameworks.

This review article highlights related progress in the field and discusses several issues on a critical path in formulating ICME using an image-based (data-driven) multiscale modeling framework with co-designed simulations and experiments. Such a framework will ultimately yield Microstructure-Statistics-Property Relations (MSPR) of highly complex nonlinear materials (see Figure 1(b)) and will lead to Virtual Materials Testing standards.

This article is organized as follows. Section 2 reviews some of the single scale models for nonlinear heterogeneous materials. Section 3 discusses multiscale modeling approaches. A particular multiscale technique, the computational theory of homogenization, is featured in Section 4 . Section 5 is devoted to an imagebased (data-driven) modeling concept. High performance computing (HPC) and parallel implementation of computational homogenization is described in Section 6. Section 7 presents model reduction, machine learning and data mining strategies for nonlinear multiscale methods. Verification, validation and uncertainty quantification in multiscale computational science and engineering are discussed in Section 8. Finally, conclusions and ongoing and expected trends and challenges are delineated in Section 9.

\section{Single Scale Models for Nonlinear Heterogeneous Materials}

Most of the progress in the past century builds on a variety of models that have been developed addressing particular physics of phenomenological descriptions at a particular scale. Any multi-scale method aims to establish a bridge between such single scale models. Single scale models may address the atomistic scale (or below), some microstructural scale, a global continuum scale or some intermediate scale. In this section, an overview is provided of the most elementary classes of single scale models, spanning a large range of the nonlinear mechanical behavior of materials. Note that each of the classes below is characterized by many underlying models, which may differ through the governing equations or approximations made to capture the physics at that scale. Characteristic time and length scales have been marked in each class.

\subsection{Density Functional Theory}

Density Functional Theory (DFT) is utilized for modeling material behavior at the smallest (subatomic) scales, and relies on the quantum-mechanical analysis of the electronic structure of many-body systems. DFT models typically span the smallest length scales $\left(<10^{-9} \mathrm{~m}\right)$ and smallest time scales $\left(10^{-15}-10^{-9} \mathrm{~s}\right)$. In this theory, the resulting properties are described on the basis of functionals of the electron density. DFT has evolved into a very popular ab initio method within the computational chemistry, condensed-matter physics, and computational physics communities. It is used to model various types of molecular interactions and to unravel the complex physical behavior at an atomistic scale. It supports the analysis of materials synthesis, processing, phase transformations, and electro-magnetical behavior. The literature on DFT within different scientific communities has become impressively large. Therefore, only a few relevant references are given here [7-12]. In the context of computational materials science, the method is particularly used to construct or assess the properties of (empirical) atomic potentials as used in molecular dynamics approaches (i.e. at a larger scale) [13-17].

\subsection{Molecular Dynamics and Atomistic models}

At the scale of atoms, molecular dynamics (MD) analyses provide direct insights into the complex mechanisms underlying the nonlinear behavior of materials at the level of single phases and interfaces. MD and atomistic models typically span the atomistic scale $\left(10^{-9} \mathrm{~m}\right)$ up to single microns $\left(10^{-6} \mathrm{~m}\right)$. Time scales are still very short $\left(10^{-12}-10^{-6} \mathrm{~s}\right)$, constituting one of the limitations of these methods. This method relies on the solution of the Newton's equations of motion for all atoms in order to extract pathdependent processes of materials. The method hinges on the underlying atomic potentials, which are used to approximate the atomic interactions [18]. A large number of potentials nowadays exist for many atomic 
systems, ranging from simple pair potentials to complex many-body potentials. Classical pair potentials are based on nearest neighbor interactions (Lennard-Jones; Morse; Torrens) [19, 20]. Tight-binding is a wellknown example of a many-body description, whereby different levels of approximation can be made (second moment, fourth moment, sixth moment, etc.). The accuracy of bond order directly depends on the accuracy of the tight-binding approximation. A third category combines properties of pair potentials and tightbonding, which is also recognized as hybrid schemes. The generalized pseudo-potential theory, the effective medium theory and the embedded atom method (EAM) fall into this category. The differences between the various existing potentials relate to their ability or accuracy to capture certain physical phenomena (e.g. ferromagnetic-paramagnetic transitions, phase transformations, energy barriers, stability, fracture, etc.). Within this context, it is worth pointing out that increasingly complex models, entailing significant computational costs, do not necessarily guarantee more physically meaningful results. This is typically the case for complex MD models, where the predictive value of any result strongly depends on the physical properties of the potential assumed, which may be incomplete in many cases.

The application of MD methods to nonlinear mechanics of materials relates to dislocations [21], microcracks and fracture [22, 23], thin films [24], surfaces, interfaces [25], etc. (see [26] for a more extensive overview). For analyzing complex microstructures, the method is rather limited in terms of the size of the domain that can be resolved. The number of atoms that can be incorporated is still limited and does not allow spanning large length scales involving billions of atoms on a regular basis. One of the largest MD simulations are those by researchers from the Lawrence Livermore National Laboratory (LLNL). In their work, a cubic piece of solid copper with an edge length of $1.56 \mu \mathrm{m}$ has been simulated using 320 billion atoms [27]. A second limitation in MD simulations relates to the time scales that can be covered, which are usually very short.

\subsection{Statistical models}

The Monte Carlo (MC) method was introduced in statistical thermodynamics in the 1950s [28]. It is now a well-know method using extensive statistical sampling to construct approximate solutions for a variety of physical/mathematical problems. Statistical models and MC methods can in principal be used at a wider range of length and time scales, depending on the physical phenomena that are treated in a statistical manner. Examples exist at atomistic length and time scales, as well as microstructural scales. The MC method establishes a probabilistic equivalent for a particular physical problem, for which the governing equations with their stochastics are transformed into integral expressions. The problem is solved by integrating these expressions using a random sampling method, which is computationally demanding. As a result, correlation functions, state equation values, kinetics, etc. can be extracted. The existing MC methods differ through their algorithmic differences in the solution and sampling strategies. For the mechanical behavior of materials, most relevant are the analyses on fracture, interfaces, diffusional processes, phase transformations, etc. [26, 29].

\subsection{Continuum models}

Many of the small scale models highlighted above (addressing single phases or interfaces) were typically used to acquire a mechanistic understanding of the different physical processes underlying the nonlinear mechanical response of materials. At the scales of complete microstructures and above (where multiple phases and interfaces co-exist), continuum models are typically used to assess material properties. Continuum models are typically used at length scales where the continuum approximation holds. This may be at the level of individual phases within a microstructure (generally larger than $10^{-5} \mathrm{~m}$ ) or macroscopic engineering scales (from $10^{-2} \mathrm{~m}$ up to $10^{+2} \mathrm{~m}$ ). The time scales in most continuum models are usually larger as well $\left(>10^{-6} \mathrm{~s}\right)$, even though impact and wave dynamics in materials may entail even smaller scales. For simplified microstructures, nonlinear micromechanics exploits continuum mechanics [30] at the level of the microstructural heterogeneities, allowing to extract macroscopic constitutive equations. This field of "continuum micromechanics" was originally established by Hill [31]. An extensive overview since then is given in [32]. Micromechanical approaches often result in closed-form analytical equations (to be solved either analytically or numerically), which clearly distinguishes them from numerical approaches where the constitutive response is computed through equilibrium simulations of the heterogeneous microstructure. 
For more complex morphologies, numerical solutions of the governing equilibrium equations in a Representative Volume Element (RVE) are more attractive [33,34]. The Representative Unit Cell (RUC) is used in the statistical mechanics community as well, for which the morphology approximation error is better quantified (i.e. statistically representative cell) using statistical descriptors [35-38] (see Section 5 for definitions of RVE and RUC and how they differ). The solution domain is generally approximated by a unit cell for periodic materials and RVEs/RUCs for statistically heterogeneous media. Proper boundary conditions need to be defined (usually representing an average strain or stress state) and a numerical solution method has to be adopted. Many of the RVEs/RUCs problems are solved using the Finite Element (FE) method, which is based on the discretization of the weak form of the governing equations. For periodic microstructural RVEs/RUCs, a spectral solver based on the Fast Fourier Transform (FTT) [39, 40] is nowadays increasingly popular. The main advantage of the latter is the high computational efficiency, which makes it popular for three-dimensional (3D) problems with many degrees-of-freedom. Unfortunately, the discrete fast Fourier transform is not easily parallelizable [41]. As presented in paper by Pekurovsky, P3DFFT overcomes a well-known scalability bottleneck of three-dimensional (3D) FFT implementations by using two-dimensional domain decomposition. However, it still achieves only $45 \%$ weak scaling efficiency. Loss of efficiency is attributed to the difficulty of maintaining scalable bisection bandwidth [42].

\subsection{Hybrid and quasi-continuum methods}

Many models at particular length scales are only partially continuum based, and discrete characteristics are naturally integrated. Hybrid and quasi-continuum models constitute a particular class in which multiple length (and time scales) are interconnected in different parts of a single domain. Therefore, they naturally span a larger range of length scales (from $10^{-9} \mathrm{~m}$ up to $10^{-2} \mathrm{~m}$ ), but the time scale range remains limited by those used at the highest spatial resolution (e.g. the atomistic region). This is for instance the case for discrete dislocation plasticity models. In other cases, complete discrete models are used as well, like microstructural lattice models or particle based models [43]. In linking length scales within a single domain, use is also made of the quasi-continuum method $[44,45]$, which recovers the discrete resolution at the smallest scale and resembles a finite element interpolation/integration at the coarse scale.

\section{Multiscale Methods for Nonlinear Heterogeneous Materials}

Modeling materials using a multiscale approach is a steadily growing field, with many past, ongoing and future developments. In this section, a compact overview is given, whereby some of the methods/approaches will be detailed in the following sections. Emphasis is put on the nonlinear response of heterogeneous materials.

The broad range of existing multiscale methods can be classified in various ways. Three typical methodological categories can be identified, differing in the manner in which the fine heterogeneous scale is incorporated [46-49]. In the first category, high resolution is applied to a small part of the domain, in which particular fine scale details of the morphology need to be resolved (e.g. dislocations, cracks, etc.). The second category relies on a macroscopic description that has to be resolved from an underlying fine scale problem (which is then probed where and when necessary). The third category is in fact a mixture of the two previous cases, where parts of the domain are fully resolved at the fine scale, and other parts are probed at the fine scale only through the effective macroscopic response. Finally, one should also note the methods relying on self-similarity across the scales [46]. A brief classification is provided here, which is however not unique. More details can be found in the literature, e.g. [48, 49]. If the methods are classified on the basis of the underlying problem formulation, the following classes can be discerned:

- Concurrent methods: Methods in which both scales are simultaneously addressed (as apparent from the problem formulation). Different length and time scales can be adopted (in a single domain) and different parts of the domain can be solved with different methodologies. In the literature, the term "concurrent" mostly refers to solution methods where different scales/methodologies are used in different subvolumes of the global domain [48]. 
- Hierarchical methods: Methods, in which the scales are linked hierarchically, i.e. different scales are resolved and coupled in the same part of a domain. The link between both scales is typically made via averaging theorems or sometimes on the basis of parameter identification only. See Section 4 for an example of this category.

- Hybrid methods: Methods revealing properties of different classes, e.g. multi-grid methods [50], quasicontinuum methods [45], wavelet-based methods, generalized finite element method [51].

If the methods are classified on the basis of the underlying algorithmic perspective (related to the actual solution procedure), the following classes can be discerned:

- Parallel methods: Methods in which both scales are solved in parallel (i.e. monolithically).

- Serial or sequential methods: Methods in which a serial algorithm is used to couple and solve both scales. The link between both scales is generally made through data passing. However, each of the scales is solved separately. Serial methods are typically hierarchical as well.

- Coupled or decoupled methods: Methods in which the solution procedure at both scales is either coupled or decoupled. Coupled schemes use on-line algorithms to resolve and couple both scales, i.e. both scales are computed during the solution of the macroscopic boundary value problem. Decoupled schemes rely on off-line computations for one of the scales, i.e. the mechanical response at this scale has been precomputed and stored for given deformation paths.

This review article will give special emphasis to a computational homogenization method, which is a hierarchical method, using an iterative (i.e. nested) coupled algorithm to connect both scales. The serial character of the method results from the data passing, which is based on the integration over small length scales. Variational methods form a special class of hierarchical multiscale methods [52, 53], where the weak form of the governing equations is split into their fine and coarse scale contributions. Note again, that different communities make use of a different terminology in addressing multi-scale methods. These methods are given attention in physics journals, mechanics journals, mathematics journals, etc. For example, the Heterogeneous Multiscale Method (HMM, $[46,54])$ is well-known in computational mathematics, whereas it is methodologically close to the computational homogenization method used in computational mechanics.

\subsection{From Micromechanics to Multi-scale Mechanics}

Multi-scale mechanics aims to identify and quantify relationships bridging various length scales in heterogeneous materials. The ultimate goal is to extract (emergent) effective/macroscopic properties at the scales of application. Adequately resolving physical, topological and statistical details of the underlying microstructure is thereby essential. Two key methods in scale bridging are "Homogenization" in the mechanics community, and "Coarse Graining" in the physics community [55, 56]. The "homogenization" method was originally coined by Ivo Babuška [57]. Homogenization essentially relies on averaging theorems. Coarse graining, on the other hand, relies on statistical mechanics/thermodynamics and aims to unravel emergent phenomena across the scales. Among these, the GENERIC framework [58-62] is one of the promising methods.

Homogenization methods are rooted in the micromechanics of heterogeneous materials. Early developments were made in the 19th century, e.g. the rule of mixtures (Voigt, 1887), the Sachs model [63], Reuss estimate (1929), Taylor model [64]. Composite materials are tailor-made multi-phase and heterogeneous materials, calling for homogenization methods in their design. This motivated the work of Eshelby [65], from which alternative continuum mechanics frameworks originated (Eshelbian mechanics; materials forces). In this early period, continuum mechanics [30] was used at the level of the heterogeneities, from which the effective macroscopic constitutive equations had to be determined. This constituted the field of "continuum micromechanics" [31], a large discipline with many contributions throughout the literature [32]. The three decades between 1950 and 1980 were marked with major progress in the homogenization of heterogeneous elastic solids, e.g. defects in elastic solids and anisotropic crystals by Kröner (e.g. [66]), the variational bounds of Hashin and Shtrikman for multiphase elastic materials (e.g. [67]), reinforced composites by Hill 
(e.g. [33]), the mean field homogenization scheme of Mori and Tanaka (e.g. [68]), interfaces by Babuška (e.g. [69]), self-consistent estimates of elastic properties for composites by Willis (e.g. [70]), etc. Extensions to nonlinear material behavior date from the early sixties and seventies (e.g. [71], [31], [72]), whereas most contributions were published in the 1980s and 1990s. Typical nonlinear phenomena studied include: plasticity and visco-plasticity, nonlinear elasticity and visco-elasticity, creep, etc. Well-known contributions were made by Nemat-Nasser (e.g. [73, 74]), Ponte Castañeda (e.g. [75]), Suquet (e.g. [76, 77]), Willis (e.g. [78]), Zaoui (e.g. [79]) and others. Evolving heterogeneities, e.g. voids in a ductile metallic matrix, were studied in the later 1970s, from which the Gurson model [80] originated. This was the start of many papers focusing on the plasticity of porous materials. The key objective was still to derive macroscopic constitutive equations that incorporate the role of the micro-scale nonlinearities. This research philosophy has been applied to study grain effects (grain-grain interaction, grain size, grain orientation/texture), inclusions/particles distributed in a hard or soft matrix with various interfaces, voids (nucleation, growth and coalescence), micro-cracks, fiber-matrix systems, etc.

The neighboring field of mathematical homogenization has played a big role in extending micromechanics to engineering applications of homogenization. Mathematical homogenization inspired engineers with good knowledge in applied mathematics to proactively apply homogenization methods to engineering problems. Historically speaking, mathematical homogenization inspired the engineering developments of the theory and applications, and also provided rigor to its foundations. The papers of J.B. Keller [81, 82], J.-L. Lions and co-workers [83, 84] and Sanchez-Palencia [85] were key in this regard. The homogenization method is now an established discipline for the analysis of heterogeneous materials. The steady evolution of advanced computational solution methods and the increase of computational power makes homogenization a strongly developed computational discipline in multi-scale mechanics.

\subsection{Nonlinear heterogeneous materials \&5 scale separation}

All engineering and physical materials are intrinsically heterogeneous at some scale (metals, polymers, composites, ceramics, biomaterials, etc.). At small scales, the behavior is generally nonlinear. Mechanisms contributing to these nonlinearities are: plastic deformation, initiating and propagating cracks, dislocation mechanics and defects, phase changes, creep and stress relaxation, microstructure evolution, etc. [32, 86-88]. Damage and fracture, governing the ultimate behavior of any materials, are typically dominated by the heterogeneous nature of the underlying microstructure. Damage spans all length scales, and is therefore one of the most difficult subjects in multi-scale mechanics. Phenomenological models exist, but real understanding is gained from analyses at heterogeneous microstructural scales, including the sub-micron scale and smaller.

Multi-scale mechanics originated from the analysis of the macroscopic behavior of heterogeneous elastic materials. Homogenization methods seek the effective response of finite statistically homogeneous materials. Materials are investigated using characteristic volumes, either periodic unit cells or RVEs/RUCs (see Section 5) [33-38]. Homogenization typically relies on a principle of separation of scales. A typical formulation thereof states that the scale of the microstructure fluctuations, $\ell_{\mu}$, must be smaller than the scale of the RVEs/RUCs, $\ell_{m}$, which again is much smaller than the macroscopic field fluctuations, $\ell_{M}$,

$$
\ell_{\mu}<\ell_{m} \ll \ell_{M} .
$$

Since its early development for elastic materials, many powerful homogenization methods have been developed for nonlinear materials, including higher-order and even nonlocal approaches, see e.g. coupled stress theory [89], Cosserat media [90], nonlocal continua [34] or higher-order elastic materials [91-93]. Physically nonlinear random microstructures have been addressed as well $[76,94,95]$, involving the Taylor-Bishop-Hill estimates, several (generalized) self-consistent schemes and asymptotic methods [96]. Incorporating both physical and geometrical nonlinearities is more challenging [97-99]. The literature on the subject extends to homogenization of nonlinear composites [100], homogenization of magneto-rheological elastomers at finite strains [101], and mathematical or asymptotic homogenization for nonlinear material behavior [99, 102-104].

One of the powerful methods developed in the past decades is the Computational Homogenization $(\mathrm{CH})$ of complex multi-phase solids [105, 106]. This method provides a nested solution of the two boundary value problems (BVPs) defined on each of the scales. The method is computationally demanding, but 
quantitatively reliable and complete, as well as easily parallelizable [107, 108]. The first-order technique (relying on a standard solid) is well-established and frequently used both in academia and industry [108120]. This paper will therefore present a more extensive summary of this particular method in what follows.

\section{Theory of Computational Homogenization}

Consider a problem involving two spatial scales: macroscopic and microscopic. The coarse macroscopic scale can be a structure, a component, or a device subjected to certain loads and constraints. At a fine (microscopic) scale, various micro-constituents are distinguished, typically presenting distinct material properties or flaws and voids (see Figure 3). This setting can be straightforwardly extended to multiple scales, however, here we will restrict ourselves to two scales only.

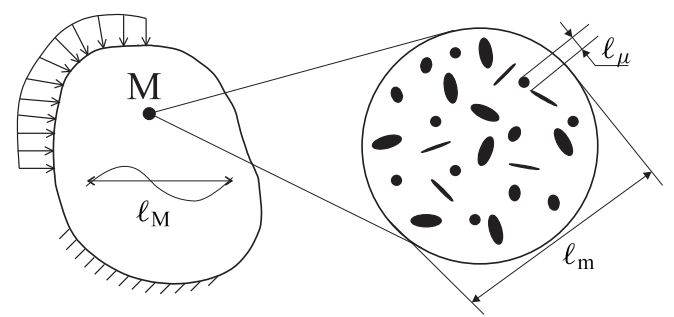

Figure 2: Length scales relevant for the separation of scales principle.

Computational homogenization, as well as most homogenization methods, critically relies on the principle of separation of scales as mentioned in Section 3. Following this definition, the absolute size of the macrostructure is, as such, irrelevant for scale separation, but the scale of the variation of the relevant macroscopic field is crucial. In many practical problems, the separation of scales defined by Eq. (1) is well satisfied. At the same time, it becomes violated in other examples, i.e. when either a micro-structural length scale tends to be large (e.g. large microstructural features, the presence of long-range correlations or percolation phenomena) or when the macro-fluctuation scale tends to be small (e.g. localization of deformation, high gradients, high frequency waves).

\subsection{Macroscale Problem}

Let the problem at the macroscopic scale be described by a field $\boldsymbol{a}_{\mathrm{M}}\left(\boldsymbol{x}_{\mathrm{M}}, t\right)$, where $\boldsymbol{x}_{\mathrm{M}}$ denotes the position vector at the macroscopic scale, typically in the reference material configuration; $t$ denotes time, which can be the physical time or a process parameter; the subscript " $M$ " denotes quantities at the macroscale, while " $m$ " will denote quantities at the microscale. The field $\boldsymbol{a}_{\mathrm{M}}$ can be a scalar, vector or tensor quantity: examples include temperature, electric or magnetic scalar potentials, or the position vector in the current configuration (related to the displacement field). The gradient of the considered field is denoted by $\mathcal{F}_{\mathrm{M}}=\boldsymbol{\nabla}_{\mathrm{M}} \boldsymbol{a}_{\mathrm{M}}$ and represents the temperature gradient, electric or magnetic field, and deformation gradient tensor, respectively, for the examples given above.

As typical in a continuum mechanics setting, the problem statement is described by a balance equation, which can be of elliptic, parabolic or hyperbolic type, with appropriate initial and boundary conditions

$$
\begin{gathered}
\nabla_{\mathrm{M}} \cdot \boldsymbol{\mathcal { S }}_{\mathrm{M}}=0, \\
\nabla_{\mathrm{M}} \cdot \mathcal{S}_{\mathrm{M}}=\alpha_{\mathrm{M}} \dot{\boldsymbol{a}}_{\mathrm{M}}, \\
\nabla_{\mathrm{M}} \cdot \mathcal{S}_{\mathrm{M}}=\alpha_{\mathrm{M}} \ddot{\boldsymbol{a}}_{\mathrm{M}},
\end{gathered}
$$

where the superimposed dot denotes the material time derivative, $\alpha_{\mathrm{M}}$ is a material constant, e.g. the heat capacity or mass density. In the above equations, $\mathcal{S}_{\mathrm{M}}$ is a constitutive quantity dual to the gradient field $\mathcal{F}_{\mathrm{M}}$. Examples of dual constitutive fields are the heat flux, electric or magnetic displacement (induction) vectors and the stress tensor. In addition, the dual constitutive field typically satisfies the Cauchy like 
relations, i.e. on a surface with outward normal $\boldsymbol{n}_{\mathrm{M}}$, it holds that $\boldsymbol{s}_{\mathrm{M}}=\mathcal{S}_{\mathrm{M}} \cdot \boldsymbol{n}_{\mathrm{M}}$, giving e.g. the surface heat flux, surface electric or magnetic induction and stress traction vector.

In order to close the above problem, constitutive relations between the constitutive field and the (history of the) gradient field are required, which are written in a general form as:

$$
\mathcal{S}_{\mathrm{M}}(t)=\mathcal{S}_{\mathrm{M}}\left\{\mathcal{F}_{\mathrm{M}}(\tau), \tau \in[0, t]\right\} .
$$

In addition, for the numerical solution of a non-linear macroscopic problem using iterative, implicit methods (e.g. Newton-Raphson scheme), a constitutive tangent $\mathcal{C}_{\mathrm{M}}$, emerging from the linearized form of the constitutive relation, is required

$$
\delta \mathcal{S}_{\mathrm{M}}=\mathcal{C}_{\mathrm{M}}: \delta \mathcal{F}_{\mathrm{M}}
$$

The main idea in computational homogenization is to obtain the constitutive closure relations for the macroscopic problem in a numerical form through the consistent construction and solution of a microstructural problem. The macroscopic and microscopic problems are thus solved in a nested manner. The computational homogenization procedure allows to directly incorporate of all relevant microstructural features and processes without the need for a priori assumptions on their effect on the macroscopic behavior. Therefore, computational homogenization removes the need for a priori mathematical formulation of the macroscopic constitutive equations and the related evolution equations, as well as parameter identification (calibration) at the macroscopic scale. Rather than identifying overall material parameters of complex multi-phase microstructures, computational homogenization relies on the knowledge (and models) of the behavior at the level of single phases and constituents. This may be well established for many materials, but still remains a challenge if a high level of accuracy or predictability is required. In the following, the microstructural boundary value problem will be formulated based on a consistent set of scale transition relations.

\subsection{Microscale Problem and Scale Transition Relations}

The microstructure for the problem at hand is described by the RVE/RUC concepts. The RVE/RUC should incorporate statistically representative details of the material morphology, but also include all the length scales relevant for the physical processes under consideration, e.g. to allow the development of percolation paths. The selection and construction of a RUC (i.e. statistically defined cell) is discussed in more detail in Section 5.

The primary field at the microscale is denoted by $\boldsymbol{a}_{\mathrm{m}}\left(\boldsymbol{x}_{m}, t\right)$ with the gradient field $\mathcal{F}_{\mathrm{m}}\left(\boldsymbol{x}_{m}, t\right)=\nabla_{\mathrm{m}} \boldsymbol{a}_{\mathrm{m}}$. In accordance with the scale separation principle (1), the RVE/RUC is infinitesimally small compared to the variations of the macroscopic fields. This implies that any temporal changes in the macroscopic fields are instantaneously accommodated at the scale of the RVE/RUC. Therefore, the RVE/RUC can be described by the stationary balance equation

$$
\nabla_{\mathrm{m}} \cdot \mathcal{S}_{\mathrm{m}}=0 \text { in } V_{\mathrm{m}}
$$

where $V_{\mathrm{m}}$ denotes the volume of RVE/RUC domain.

The constitutive behavior relevant for the problem of interest is assumed to be known for each microstructural constituent $\iota$, as well as the laws for their interaction, interface behavior, etc.

$$
\mathcal{S}_{\mathrm{m}}^{(\iota)}(t)=\mathcal{S}_{\mathrm{m}}^{(\iota)}\left\{\mathcal{F}_{\mathrm{m}}^{(\iota)}(\tau), \tau \in[0, t] ; \xi_{\mathrm{m}}^{(\iota)}\right\}
$$

where $\xi_{\mathrm{m}}^{(\iota)}$ denotes a set of internal state variables, required to describe the relevant microstructural evolution processes. Note that (in $\mathrm{CH}$ ) internal variables do not appear explicitly in the macroscopic constitutive relation Eq. (5), since all the relevant internal variables are contained within the microscale problem. As a side remark, it should be pointed out that identification and characterization of constitutive behavior at the fine scale is in general not a trivial problem and might require complicated in situ measurements.

To complete the microscale problem Eqs. (7)-(8), boundary conditions need to be specified. In the computational homogenization framework, boundary conditions for the microscale problem are formulated through scale transition relations that establish a relationship between the macro- and microscale problems. To this aim, the microscopic field $\boldsymbol{a}_{\mathrm{m}}\left(\boldsymbol{x}_{\mathrm{m}}, t\right)$ is written as a superposition of the macroscopic homogeneous 
part, given by the macroscopic gradient field $\mathcal{F}_{\mathrm{M}}(t) \equiv \mathcal{F}_{\mathrm{M}}\left(\boldsymbol{x}_{\mathrm{M}}, t\right)$ at the macroscopic point for which the microscopic problem is being constructed, and a micro-fluctuation field $\boldsymbol{w}_{\mathrm{m}}\left(\boldsymbol{x}_{\mathrm{m}}, t\right)$, which represents the local variation of the microscale field due to the presence of the heterogeneous microstructure

$$
\Delta \boldsymbol{a}_{\mathrm{m}}\left(\mathbf{x}_{\mathrm{m}}, t\right)=\mathcal{F}_{\mathrm{M}}(t) \cdot \Delta \boldsymbol{x}_{\mathrm{m}}+\boldsymbol{w}_{\mathrm{m}}\left(\boldsymbol{x}_{\mathrm{m}}, t\right),
$$

where $\boldsymbol{x}_{\mathrm{m}}$ is the position vector of the points within the RVE/RUC in the reference (material) configuration; $\Delta$ denotes the relative value of a field with respect to an arbitrary reference point. Computing the microscopic gradient field $\mathcal{F}_{\mathrm{m}}$ from (9) gives

$$
\mathcal{F}_{\mathrm{m}}\left(\boldsymbol{x}_{\mathrm{m}}, t\right)=\boldsymbol{\nabla}_{\mathrm{m}} \boldsymbol{a}_{\mathrm{m}}=\mathcal{F}_{\mathrm{M}}(t)+\boldsymbol{\nabla}_{\mathrm{m}} \boldsymbol{w}_{\mathrm{m}}\left(\boldsymbol{x}_{\mathrm{m}}, t\right) .
$$

Next, the first scale transition relation is postulated, requiring the RVE/RUC volume average of the microscopic gradient field to be equal to its macroscopic counterpart

$$
\mathcal{F}_{\mathrm{M}}(t)=\frac{1}{V_{\mathrm{m}}} \int_{V_{\mathrm{m}}} \mathcal{F}_{\mathrm{m}}\left(\boldsymbol{x}_{\mathrm{m}}, t\right) \mathrm{d} V_{\mathrm{m}} .
$$

Upon substitution of (10) into (11), it is clear that the scale transition relation (10) is automatically satisfied if the volume averaged contribution of the gradient of the micro-fluctuation field vanishes

$$
\frac{1}{V_{\mathrm{m}}} \int_{V_{\mathrm{m}}} \nabla_{\mathrm{m}} \boldsymbol{w}_{\mathrm{m}} \mathrm{d} V_{\mathrm{m}}=\frac{1}{V_{\mathrm{m}}} \int_{A_{\mathrm{m}}} \boldsymbol{n}_{\mathrm{m}} \otimes \boldsymbol{w}_{\mathrm{m}} \mathrm{d} A_{\mathrm{m}}=\mathbf{0},
$$

where the Gauss divergence theorem has been applied with $A_{\mathrm{m}}$ being the RVE/RUC outer boundary and $\boldsymbol{n}_{\mathrm{m}}$ representing the outward normal vector. Condition (12) can be fulfilled a priori by prescribing appropriate boundary conditions on the micro-fluctuation field $\boldsymbol{w}_{\mathrm{m}}$. The following options are typically used in the literature:

- fully prescribed essential boundary condition: prescribe $\boldsymbol{w}_{\mathrm{m}}=0$ for all points on the RVE/RUC boundary, i.e. not allowing for any micro-fluctuations on the RVE/RUC boundary;

- periodic boundary conditions: for a RVE/RUC of an initially periodic shape, i.e. the normals are opposite on the '+' and '-' sides of the RVE/RUC boundary, $\boldsymbol{n}_{\mathrm{m}}^{+}=-\boldsymbol{n}_{\mathrm{m}}^{-}$, require the periodicity of the micro-fluctuation field $\boldsymbol{w}_{\mathrm{m}}^{+}=\boldsymbol{w}_{\mathrm{m}}^{-}$;

- minimal essential boundary condition: impose the constraint Eq. $(12)_{2}$ directly requiring the integral over the entire RVE/RUC boundary to vanish.

Note that the Voigt/Taylor averaging model requiring a uniform gradient field in all microstructural constituents is recovered if the micro-fluctuation field vanishes, $\boldsymbol{w}_{\mathrm{m}}=0$, in the entire RVE/RUC volume, which trivially satisfies Eq. (12). The periodic boundary conditions are frequently used in practice, since they have been shown to provide faster convergence towards the effective material properties upon increasing the size of the microstructural computational cell (i.e. including more microstructural features) $[115,121-125,125-$ 128]. The fully prescribed essential boundary conditions are also often used, mostly due to the simplicity of their implementation. Minimal kinematic boundary conditions have also been employed [127, 128]. Finally, more advanced types of RVE/RUC boundary conditions have recently been developed for specific problems, e.g. strain localization $[129,130]$.

With the boundary conditions established, the RVE/RUC boundary value problem can be readily solved using any available (numerical) technique, e.g. finite differences, finite elements, spectral methods, etc. Upon the solution of the microstructural problem, another scale transition relation is needed to upscale the microscopic constitutive response to the macroscale. In computational homogenization, the scale transition 
relation is usually established by requiring equality between the local macroscopic generalized power (per unit volume) and the volume averaged generalized power of the corresponding RVE/RUC,

$$
\mathcal{S}_{\mathrm{M}}: \dot{\mathcal{F}}_{\mathrm{M}}=\frac{1}{V_{\mathrm{m}}} \int_{V_{\mathrm{m}}} \mathcal{S}_{\mathrm{m}}: \dot{\mathcal{F}}_{\mathrm{m}} \mathrm{d} V_{\mathrm{m}} .
$$

In mechanics of materials, this relation is known under the name Hill-Mandel Lemma or macro-homogeneity condition (see Hill [33] and Suquet [109]). Using the fact that $\mathcal{F}$ is the gradient quantity, applying the chain rule and the Gauss theorem to (13), allows establishing the relation between the microscopic and macroscopic constitutive quantities

$$
\mathcal{S}_{\mathrm{M}}=\frac{1}{V_{\mathrm{m}}} \int_{V_{\mathrm{m}}} \mathcal{S}_{\mathrm{m}} \mathrm{d} V_{\mathrm{m}}=\frac{1}{V_{\mathrm{m}}} \int_{A_{\mathrm{m}}} \boldsymbol{s}_{\mathrm{m}} \otimes \boldsymbol{x}_{\mathrm{m}} \mathrm{d} A_{\mathrm{m}},
$$

with $\boldsymbol{s}_{\mathrm{m}}=\mathcal{S}_{\mathrm{m}} \cdot \boldsymbol{n}_{\mathrm{m}}$ being the surface flux. The last expression in Eq. (14) shows that the macroscopic constitutive quantity can be efficiently computed from only the microscale RVE/RUC boundary fluxes.

Computing $\mathcal{S}_{\mathrm{M}}$ from the microscale RVE simulations at each time instance therefore provides the macroscopic constitutive response, Eq. (5), in a numerical form. To obtain the constitutive tangent defined by Eq. (6), different techniques can be used, e.g. numerical differentiation. Although easy to execute, numerical differentiation is not efficient since it requires additional RVE/RUC simulations, which are usually expensive. Alternatively, more efficient techniques are based on the Schur complement of the microscopic linearized system of equations (see $[117,122]$ for more details on this procedure in combination with direct solvers). For parallel iterative solvers, procedures developed in $[107,108]$ can be employed.

Finally it should be noted that in some problems, the constitutive dual quantity depends on both the gradient of the primary field and on the absolute value of the primary field itself. For example, many materials have temperature dependent material properties. In this case, an additional scale transition relation has to be formulated. For example, in [131, 132] the thermal energy consistency condition was enforced by requiring the volume average of the RVE/RUC stored heat to be equal to the local macroscopic stored heat (per unit volume). After elaboration, this leads to an additional constraint to be imposed on the microscopic temperature field.

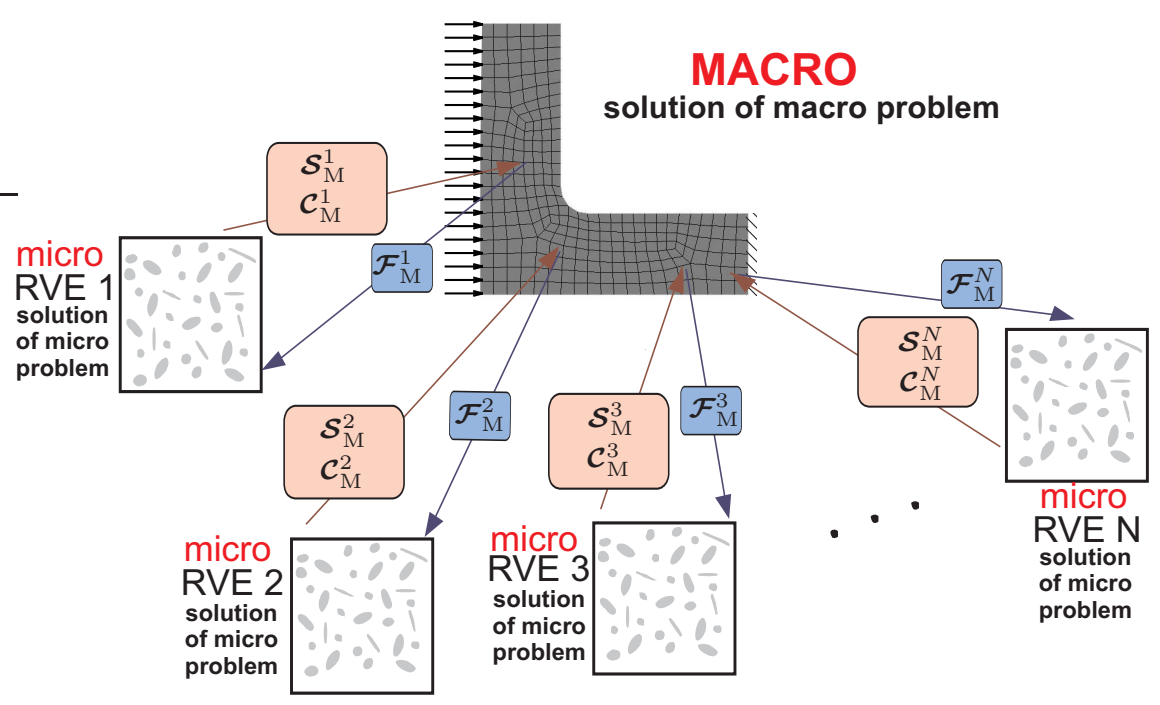

Figure 3: Computational homogenization scheme.

The computational homogenization framework is schematically illustrated in Figure 3. The macroscopic problem is typically solved in a discretized form by any available numerical technique, e.g. finite differences, 
finite elements, spectral methods, etc. The macroscopic gradient $\mathcal{F}_{\mathrm{M}}$ is computed at every sampling or integration point of the macrostructure. Next, $\mathcal{F}_{\mathrm{M}}$ is used to formulate the boundary conditions imposed on the RVE/RUC assigned to this point. Upon solving the microscopic boundary value problem, the macroscopic dual constitutive quantity $\mathcal{S}_{\mathrm{M}}$ is obtained, thus providing the numerical constitutive closure relationship at the macroscopic point. The local macroscopic constitutive tangent $\mathcal{C}_{\mathrm{M}}$, which is needed for the implicit iterative solution of the macroscopic non-linear problem, is also derived from the microstructural analysis.

The implementation and application of the computational homogenization scheme as described above is straightforward and highly modular, i.e. different combinations of numerical techniques and software codes can be used. However, the computational costs, both CPU time as well as memory requirements are typically high. These can be substantially alleviated through a high performance parallel implementation and/or through the application of reduced order modeling concepts. These two aspects will be discussed subsequently in sections 6 and 7 , respectively.

\subsection{Transient Effects}

The computational homogenization scheme described above is applicable to stationary, as well as transient problems. In the latter case, however, only the transient problems satisfying the scale separation condition Eq. (1) can be considered directly, i.e. when the characteristic macroscopic wave-length is much larger compared to all the relevant microstructural length scales. In this case, it can be assumed that any temporal changes at the macroscale are instantaneously accommodated at the microscale. Thus, the microscale can be considered stationary, described by Eq. (7), with the boundary conditions emanating from Eq. (11) as discussed above. The material constant related to the transient term, e.g. heat capacity or mass density can be obtained by volume averaging of the corresponding microscopic quantity

$$
\alpha_{\mathrm{M}}=\frac{1}{V_{\mathrm{m}}} \int_{V} \alpha_{\mathrm{m}} \mathrm{d} V_{\mathrm{m}} .
$$

In this form, computational homogenization has been successfully applied to heat conduction $[131,132]$ and mechanical problems $[102,133]$ for example.

For cases in which the macroscopic transient phenomena interact with the microscale transient response, e.g. high frequency waves or wave dispersion, the full scale separation is not valid and basic computational homogenization as described above may not be applicable. In some particular cases, however, a relaxed separation of scales holds, such that the microscale transient response (e.g. micro-inertia) is localized only in some of the micro-constituents embedded in a matrix (host) material, for which the full separation of scales may apply. This is, for example, the case for so-called locally resonant acoustic metamaterials, containing resonators (e.g. a mass on a spring) embedded in a stiff matrix material. This case has recently been treated by an extended version of the transient computational homogenization [134], where the effect of micro-inertia naturally emerges at the macroscopic scale through an enriched field, with an additional macroscopic balance equation [135]. Finally, we note that multiscale modeling of temporal scales is an active area of research for $\mathrm{CH}$ and concurrent multiscale methods alike.

\subsection{Applications and Extensions}

The computational homogenization scheme as described above has initially been developed and applied for purely mechanical non-linear quasi-static problems [109-111, 113-118, 122, 136, 137]. Since then, the computational homogenization approach has been adapted to problems involving other fields, as well as coupled multi-physics problems, e.g. thermal [131, 138], thermo-mechanical [132], electro-magneto-mechanical [139-143], diffusion [144], liquid-phase sintering [145], and problems involving chemical couplings [146]. A limited selection of problems to which computational homogenization has been applied includes: porous media [147-150], cellular materials [151, 152], polycrystalline metals [153, 154], technical textiles [155], granular materials [156], reinforced elastomers [99], trabecular bone [157], biological tissue [158] and Li-ion battery cells [159]. 
In addition, several modified and extended computational homogenization schemes have been developed to go beyond the limitations of the classical (also called first-order) framework. For example, second-order computational homogenization for mechanical problems couples standard (first-order) continuum microstructures to the higher-order gradient continuum at the macroscale [160-166]. This approach slightly relaxes the separation of scales and allows treatment of moderate localization of the macroscopic field and captures the influence of the absolute size of the microstructure into the homogenization scheme which is otherwise insensitive to it. A more advanced version of the higher-order computational homogenization, which relaxes the constraints on higher-order continuity requirements and boundary conditions is based on the computational continua approach $[167,168]$.

Moving towards strong localization of macroscopic fields is a challenging topic in computational homogenization due to the lack of separation of scales [169-171]. One extension to remedy this problem that is gaining interest is the computational homogenization of interfacial volumes towards cohesive zones [120] for damage and fracture. Unlike the standard first-order technique, this scheme can properly handle localization at the macroscopic scale. For bulk homogenization, localization of macroscopic fields has recently been addressed in continuous-discontinuous homogenization-localization approaches. In this case, the microscale properties within the localization band are directly upscaled to the macroscale, the description of which is enriched by either a discrete band (weak discontinuity) $[172,173]$ or a discontinuous jump (strong discontinuity) [174-177] representing the propagating fracture at the macroscale.

Another class of computational homogenization approaches involves dimensional reduction in addition to homogenization. For example, computational homogenization applied to beams, plates, and shells considers microstructural models having the thickness corresponding to the physical thickness of the shell; in-plane homogenization is then performed, combined with through thickness integration [178-180]. Application of computational homogenization to heterogeneous interfaces and (adhesive) layers typically couples a (zero thickness) cohesive zone type description at the macroscale to an RVE/RUC at the microscale. This technique fully resolves the microstructure of the layer as well as damage and fracture within it [107, 108, 120, 181-184]. Finally, another interesting application of computational homogenization involves contact and friction problems allowing an efficient solution of these naturally multi-scale non-linear problems [185-187].

\section{Image-based Materials Modeling}

When modeling geometric and material nonlinearities in heterogeneous materials, analyzing realistic microstructures is critical for achieving predictive capabilities. Heterogeneous materials may contain a variety of heterogeneous phases (voids, inclusions, fibers, crystals, etc.) of varying composition, size and shape with complex spatial configuration. Statistically preserving this morphology in a representative computational domain is important. The image-based (data-driven) modeling concept focuses on obtaining detailed threedimensional data sets from state of the art imaging methods, e.g., micro- and nano-computed tomography (CT) or scanning electron microscopy (SEM), and constructing optimal RUCs for further investigation of transport, mechanical, optical, electromagnetic and other properties. Moreover, image-based multiscale modeling with co-designed simulations and experiments within the integrated computational materials engineering framework is paving the way for predictive materials modeling to become reality (see Section 1).

Selection of the imaging method is often determined by the material composition, e.g. density, electromagnetic wave absorption/reflection properties, and length scale of heterogeneities. CT (both micro-CT and nano-CT [191]) is a particularly attractive imaging method, as it allows for three-dimensional representations to be obtained nondestructively. Nondestructive techniques also allow for in situ testing and enable micro/nano-scale validation (see Section 8). Some examples of complex material systems imaged with micro-CT and a dual focused ion beam and scanning electron microscope (FIB/SEM) are shown in Figure 4. Commercial CT machines with X-ray tube sources can be used to characterize materials with moderate densities and volumes on the $\mathcal{O}(\mathrm{mm})$ containing $\mathcal{O}\left(10^{5}\right)$ geometric objects, and applications include solid rocket propellants [192, 193], glass beads [38, 194], paper [195], and engineered cementitious composites [196]. For denser material such as metals, CT methods require high intensity electromagnetic radiation generated from 


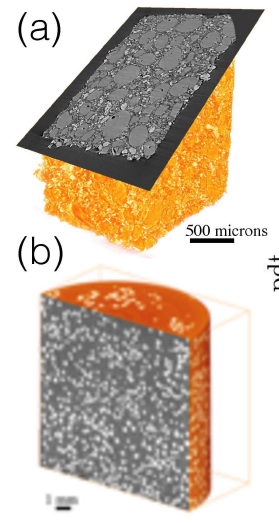

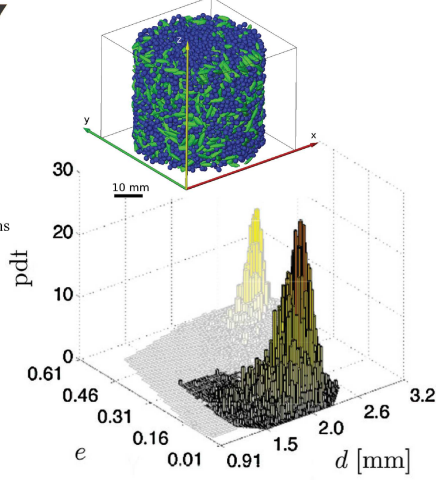

(c)
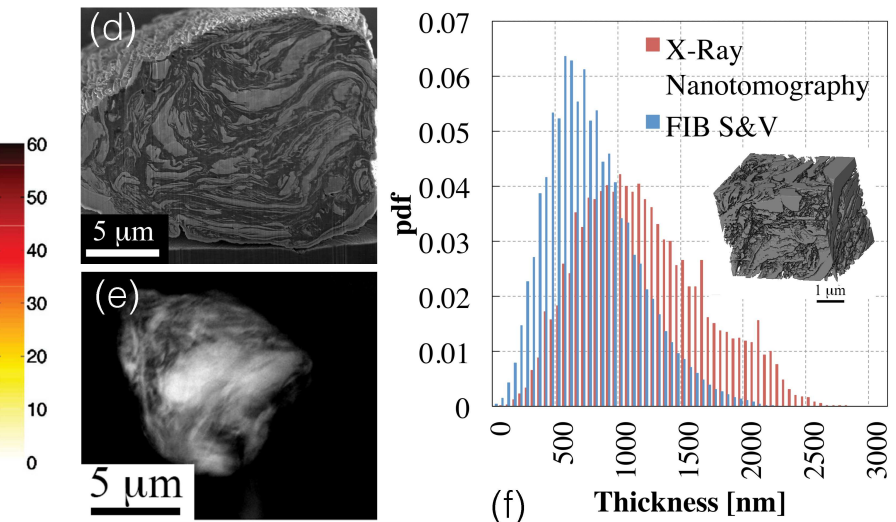

(f)

Thickness [nm]

Figure 4: Three-dimensional imaging techniques for heterogeneous materials. (a-c) Three-dimensional renderings of particulate composites using micro-CT: (a) solid rocket propellant (from Lee et al. [188]), (b) silica-reinforced rubber (from Computational Physics Group (CPG) at the University of Notre Dame), and (c) granular system of polydisperse ellipsoids/spheres (from Gillman et al. [189]) and associated multi-dimensional particle size and shape distribution. In the particle size distribution, the variables $d$ and $e$ are the representative sphere diameter and eccentricity, respectively. (d) Cross sectional image from FIB/SEM of binary metal composite (Nickel-Aluminum) synthesized through high energy ball milling. (e) Projection image of $\mathrm{Ni}$-Al metal composite using nano-CT beamline at Argonne National Laboratory's Advanced Photon Source. (f) The thickness distribution of the bicontinuous material is compared for (d) FIB/SEM and (e) nano-CT imaging/characterization methods. ((d), (e), and (f) from Shuck et al. [190]).

synchrotron sources $[190,197]$, and access to these facilities can be limited. Alternatively, serial sectioning techniques such as dual beam FIB/SEM systems are commonly used for 3D characterization of dense materials, but this technique is limited by its destructive nature and low analysis speed. Serial sectioning techniques have been used for imaging polycrystalline metals [198], metal alloys [199], high energy ball milled metal powders [190], and fuel cells [200]. Microstructural-crystallographic orientations are often studied by the Electron Backscatter Diffraction (EBSD) technique for crystalline or polycrystalline materials [201]. For biological systems, magnetic resonance imaging (MRI) and diagnostic sonography (ultrasonography) are popular in Patient-specific Modeling [202].

\subsection{Statistically Representative Unit Cells}

Despite the ability of today's imaging techniques to analyze large volumes of heterogeneous materials, the data sets are often too large to be completely resolved in practical numerical simulations of nonlinear material behavior. In order to perform efficient and accurate multiscale simulations, it is critical to develop a representative computational domain of the microstructure. As mentioned in Section 2, the terms RVE and RUC are two common terms designating computational domains that preserve important geometric features and also accurately capture the macro- and micro-scale fields. Hill [33] proposed a definition of an RVE to be a volume of material that is large enough to represent a whole ensemble of microstructures in an average sense and contains a sufficient number of heterogeneities to eliminate boundary effects (for boundary values that are macroscopically uniform). Drugan and Willis [34] introduced a variant RVE definition, where it is the smallest material volume element that accurately captures the mean constitutive response. Povirk [35] considered yet another variant with emphasis on preserving the statistical description of the microstructure. In [35], a RUC is denoted as an optimally sized domain that preserves the statistical description of the original microstructure. In all of these definitions, the RVE should be both statistically representative (i.e. preserves geometrical complexity of heterogeneous microstructure) and physically meaningful (i.e. accurately predicts effective material behavior). These two requirements are mutually inclusive, as determining the overall behavior requires accurate characterization of the morphology [203, 204]. It is important to note that a perfect RVE (i.e. one that would fully satisfy a scale separation relation) is only realized in the infinite volume limit, but an optimally constructed RUC results in effective material predictions with statistically quantifiable errors [181]. Thus, we focus on RUC construction and use RUC nomenclature in what follows. 
Preserving first order measures such as the volume fraction and sizes of crystals, particles, and ligaments (see Figures 4(c) and (f)) in a RUC is typical for many studies in multiscale modeling [205-209]. In these first order theories, the spatial distribution of micro-heterogeneities plays a secondary role in accurate modeling, and is often neglected. However, reconstruction techniques that preserve higher order correlations result in optimally sized RUCs while reducing errors in estimates of overall macroscopic properties $[2,181,203,204$, 210, 211]. Note that importance of higher order statistical information depends on the property of interest that is being investigated (e.g. Young's modulus versus fracture toughness). The random complex nature of heterogeneous microstructures makes volume averaged statistical descriptors well suited for quantifying the spatial arrangement (distribution) of phases. Many statistical descriptors have been proposed to quantify the complex random morphologies in heterogeneous materials (see the book by Torquato [212] for example).

In order to construct an optimal RUC that preserves the statistical makeup of large composite microstructures, an optimization problem that minimizes differences of statistical descriptors between real and synthetic microstructures is typically formulated. In this regard, work utilizing two-point probability functions as well as other statistical descriptors (e.g. the lineal path function, the power spectral density, a stress interaction parameter) and their combination has been performed [35-38, 211, 213-220]. In many of these works, the pixel or voxel-based representation is popular for statistical characterization and synthetic structure generation. Although relatively simple, this representation distorts geometrical shapes, i.e. any object (a rhombus or an ellipsoid) can be approximated by a cluster of voxels, but the surface description is highly pixel or voxel dependent (regardless of the resolution). Thus, the voxel-based representation is inappropriate for surface driven phenomena, such as a particle decohesion, surface diffusion, and many others. Thus, algorithms that preserve the spatial distribution as well as the shape and size of heterogeneities in three-dimensions are preferred and yield RUCs for optimal simulation of many physical phenomena [38, 189].

As noted above, a common measure utilized in generating RUCs is the $n$-point probability function. The $n$-point probability function is defined as the ensemble average (denoted by an overbar) of the indicator function, $\chi_{q}\left(\vec{x}_{1}\right)$, for $n$ points:

$$
S_{q s \cdots t}\left(\vec{x}_{1}, \vec{x}_{2}, \cdots, \vec{x}_{n}\right)=\overline{\chi_{q}\left(\vec{x}_{1}\right) \chi_{s}\left(\vec{x}_{2}\right) \cdots \chi_{t}\left(\vec{x}_{n}\right)} .
$$

$S_{q s \cdots t}\left(\vec{x}_{1}, \vec{x}_{2}, \cdots, \vec{x}_{n}\right)$ is the probability of observing phases $q, s, \cdots, t$ at points $\vec{x}_{1}, \vec{x}_{2}, \cdots, \vec{x}_{n}$, respectively. Analytical expressions of the $n$-point probability functions are difficult to formulate for random materials. Therefore, Monte Carlo-based statistical sampling algorithms are frequently used. A spherical sampling template is employed in work by Coker and Torquato [221, 222] and Lee et al. [38]. A more advanced adaptive sampling strategy has been presented in [203, 204]. While these functions are powerful for quantifying the spatial configuration of the microstructure, they can also be utilized directly in micromechanics for both linear [188, 189, 203, 212, 223] and nonlinear [224, 225] regimes.

Optimization problems are typically formed to reconstruct a RUC that reflects the statistical character of large volumes obtained through imaging techniques. An objective function, $f$, is formulated to quantify the differences in relevant statistical descriptors, $\mathcal{S}_{i}$, between the image-based data set (denoted by superscript $p$ ) and a RUC (denoted by superscript $c$ ), and is defined as

$$
f(\overrightarrow{\mathcal{X}})=\sum_{i}^{N_{s}} \beta_{i}^{s} \mathcal{F}_{i}+\sum_{i}^{N_{p}} \beta_{i}^{p} \mathcal{P}_{i}, \mathcal{F}_{i}=\left|\mathcal{S}_{i}^{p}-\mathcal{S}_{i}^{c}\right| .
$$

Here, $\overrightarrow{\mathcal{X}}$ is the set of physically admissible geometric parameters subject to minimization, and $\mathcal{P}_{i}$ are penalty functions to enforce geometric constraints, e.g. impenetrability of inclusions. $N_{s}$ and $N_{p}$ are the number of statistics functions and number of penalty functions, respectively, and $\beta_{i}^{s}$ and $\beta_{i}^{p}$ are weights for each term in the objective function.

An example of a reconstruction procedure for particulate systems is presented here from [38], where $n$-point probability functions are considered. In [38], two separate objective functions are minimized inde- 


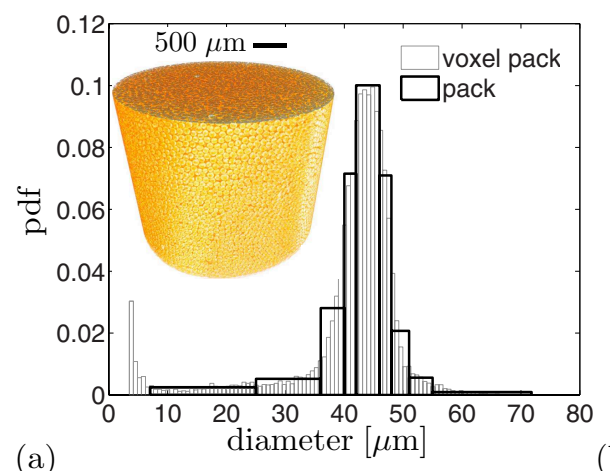

(a)

(b)

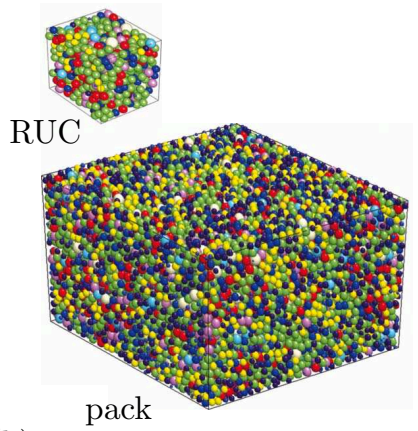

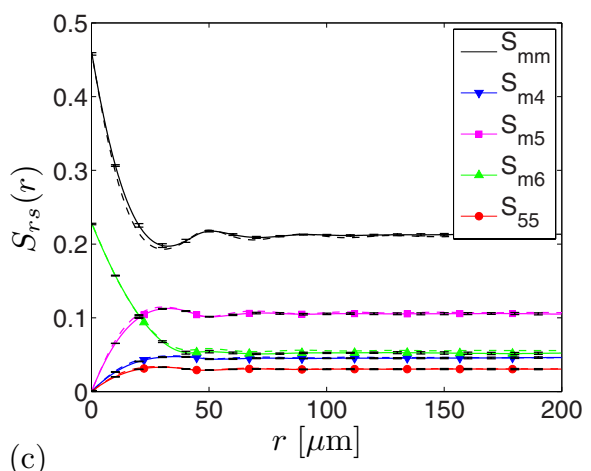

(c)

Figure 5: RUC reconstruction example based on minimizing differences in one- and two-point probability functions for a granular pack of silica. (a) Particle size distribution from tomographic characterization (see inset image). (b) Pack and a reconstructed RUC. (c) Comparison of two-point probability functions between the pack (dashed lines) and 5 reconstructed RUCs (solid lines). Here solid lines represent the mean over the 5 realizations, and error bars indicate the standard deviation. Note that the tomographic data is called the "voxel pack", and the cell generated based on the voxel data by replacing the inclusions with spheres is called the "pack". (from Lee et al. [38]).

pendently. A first objective function for an $N$-phase material is defined as

$$
\mathcal{F}_{1}\left(\ell_{\text {cell }}\right)=\sqrt{\sum_{q}^{N}\left(c_{q}^{p}-c_{q}^{c}\right)^{2}}=\sqrt{\sum_{q}^{N} c_{q}^{p}-\frac{V_{q} n_{q}{ }^{2}}{\ell_{\text {cell }}^{3}}},
$$

where $c_{q}^{p}$ and $c_{q}^{c}$ are the volume fractions for material phase $q . V_{q}$ and $n_{q}$ are the volume and the number of heterogeneous phases (i.e. geometric entities: particles, fibers, voids, etc.) in the cell for phase $q$. The saturation point of the two-point probability function $\left(r_{\text {sat }}\right)$ provides an adequate initial guess, $\ell_{\text {stat }}=2 r_{\text {sat }}$, for the side length of the unit cell, $\ell_{\text {cell }}$. Next, minimization of this function yields the geometrical size of the cell, $\ell_{\text {cell }}$, that best preserves volume fractions (first-order statistics). A second objective function is introduced to determine the spatial distribution of the constituents. This function is defined as

$$
\mathcal{F}_{2}\left(\vec{x}_{n}\right)=\sum_{q}^{N} \sum_{s}^{N}\left\|S_{q s}^{p}-S_{q s}^{c}\right\|_{L_{2}}
$$

and represents the $L_{2}$ error in the two-point statistics, $S_{q s}$. Here, $\vec{x}_{n}$ are the coordinates of the heterogeneities in the cell. A penalty function to enforce impenetrability of inclusions is also imposed [38].

In [38], this reconstruction technique is performed for a tomographically characterized granular system composed of polydisperse silica (see inset of Figure 5(a)). A large section (pack, see Figure 5(b)) from the image data set $\left(1445.37 \times 1287.89 \times 789.11 \mu \mathrm{m}^{3}\right)$ is considered that contains 19,892 particles. The polydispersity of this system is reflected in the particle size distribution (see Figure 5(a)), and the distribution is discretized into 9 distinct sizes (bins/modes) for further analysis. As mentioned above, the saturation point of the two-point probability function $\left(r_{\text {sat }}\right)$ provides a good estimate for an optimally sized RUC, $\ell_{\text {cell }}$. For the granular system in Figure $5(\mathrm{a})$, the statistical length scale is determined to be $\ell_{\text {stat }}=2 r_{\text {sat }}=400 \mu \mathrm{m}$ ( 10 mean particle diameters). Utilizing this knowledge, five RUCs of the second statistical order were reconstructed using a genetic algorithm [37, 215]. One of these RUCs is shown in Figure 5(b), and all RUCs contain 1082 particles with $\ell_{m}=\ell_{\text {cell }}=399.6 \mu \mathrm{m}$ (geometric length scale, see Eq. (1)). Figure 5(c) shows the good agreement achieved in the two-point statistics. Note the small standard deviation for 5 cell realizations.

\subsection{Impact of Microstructure on Multiscale Simulations}

As introduced by Hill, Drugan and Willis, and Povirk, preserving the important geometric features is one critical aspect in accurately modeling heterogeneous materials. Utilizing the statistical reconstruction 
framework described above, the connection between a geometric material length scale, $\ell_{\text {cell }} \approx \ell_{\text {stat }}$, and the optimally sized RUC, $\ell_{\mathrm{RUC}}$, is illustrated by determining the effective nonlinear material behavior in progressively damaged microstructures.
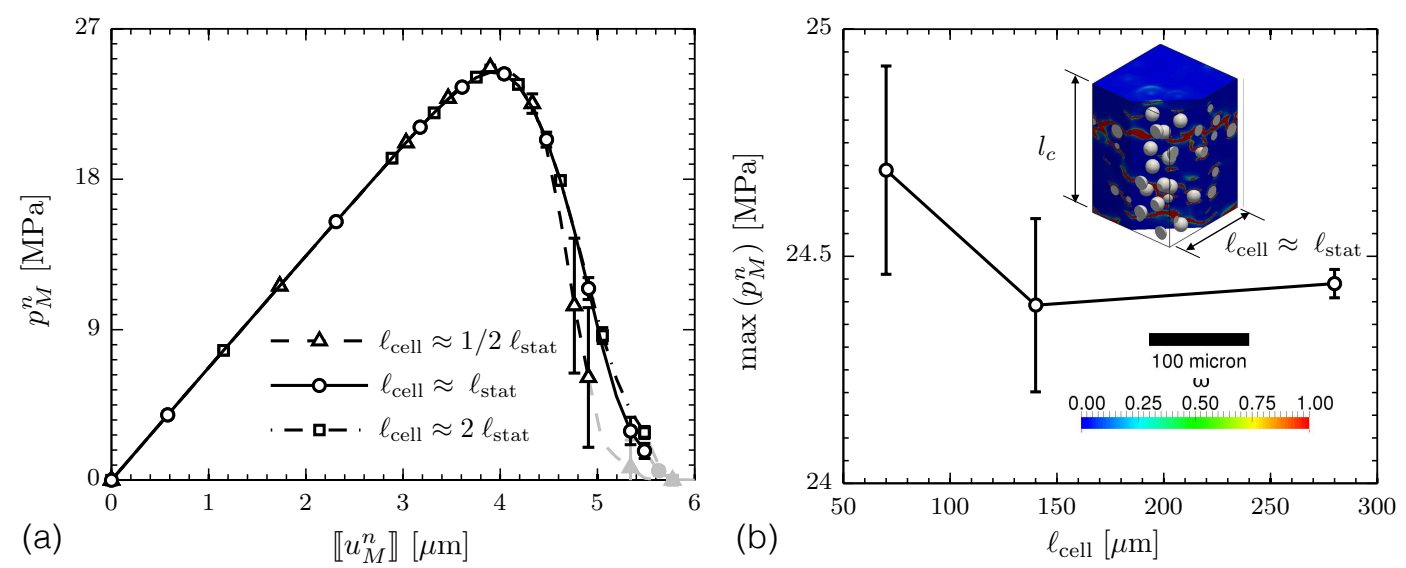

Figure 6: Effect of cell size on macroscopic traction-separation response. Note that the statistical length scale for this material is $\ell_{\text {stat }}=140 \mu \mathrm{m}$. (a) Comparison of the average normal traction-separation response for different sized cells. (b) Convergence of the normal strength with increasing cell size. The inset image shows the damage field, $\omega \in(0,1)$, for a $\ell_{\text {cell }} \approx \ell_{\text {stat }}$ cell. Figures taken from Mosby and Matouš [181]. (c) IOP Publishing. Reproduced with permission. All rights reserved.

An example from [181] is considered, where the damage response of three different sized cells is simulated in the context of multiscale cohesive modeling [120] of heterogeneous adhesive layers. Here, micro-scale failure is modeled in a 3D finite strain setting with a viscous isotropic damage model (see [120, 226, 227] on constitutive damage theory). This damage process introduces a material (physical) length-scale, $\ell_{\mu}$, that represents diffusive thickness of micro-cracks (see inset image in Figure 6(b)). Five statistically (macroscopically) equivalent RUCs of different morphology (microstructure) for three cell size were reconstructed. Note that for multiscale cohesive modeling, the height of the cell, $l_{c}$, is fixed and the side length of the unit cell, $\ell_{\text {cell }}$, is the in-plane cell dimension (see Mosby and Matouš [181]). The heterogeneous RUCs with a particle volume fraction of $c_{p}=0.1$ contain 23,93 , and 374 particles of diameter $10 \mu \mathrm{m}$ for cell side lengths of $\ell_{\text {cell }} \approx 1 / 2 \ell_{\text {stat }}, \ell_{\text {cell }} \approx \ell_{\text {stat }}$, and $\ell_{\text {cell }} \approx 2 \ell_{\text {stat }}$, respectively.

The macroscopic traction-separation response is shown in Figure 6(a), where the error bars represent one standard deviation over 5 cell realizations. Here, $p_{M}^{n}$ and $\llbracket u_{M}^{n} \rrbracket$ are the normal macroscopic first PiolaKirchhoff traction and displacement jump vectors at the interface (across the height, $l_{c}$ ), respectively. When comparing the macroscopic response, the hyper-elastic behavior is nearly identical for all cell sizes. This is in agreement with analysis of linear modeling, where it has been demonstrated that a limited number of inclusions $(\sim 12-30)$ is sufficient for capturing the mean response [34, 40, 205, 228]. However, as illustrated here for nonlinear material behavior involving damage and material softening, $\mathcal{O}\left(10^{2}\right)$ inclusions are required to capture the mean response of the complete loading history for even moderate particle volume fractions [181]. Moreover, the mesh resolution needed to resolve $\ell_{\mu}$ was determined by a convergence study and Richardson extrapolation [229], and indicated $h_{\text {mean }} \leq 1.5 \mu \mathrm{m}$. These requirements of a large number of inclusions (to capture the morphology, $\ell_{\mathrm{RUC}}$ ) and small numerical discretization (to capture the physics, $\ell_{\mu}$ ) lead to very large computations. Therefore, an efficient parallel solution strategy is essential (see Section 6).

The coupling between the strong nonlinearity and the microstructure affects the limit point and softening responses. While the two larger unit cells $\left(\ell_{\text {cell }} \approx \ell_{\text {stat }}\right.$ and $\left.\ell_{\text {cell }} \approx 2 \ell_{\text {stat }}\right)$ have small and overlapping error bars as the material softens, the smallest cell $\left(\ell_{\text {cell }} \approx 1 / 2 \ell_{\text {stat }}\right)$ has a distinctly different response with large standard deviation. One important quantity of interest is the maximum first Piola-Kirchhoff traction (strength). The convergence of the normal strength with respect to the side length of the unit cell, $\ell_{\text {cell }}$, is displayed in Figure 6(b). This figure shows the rapid convergence of both mean and standard deviation with increasing cell size, and confirms the connection between $\ell_{\mathrm{RUC}}$ and $\ell_{\text {cell }} \approx \ell_{\text {stat }}$.

It is important to close with the understanding that the material, $\ell_{\mu}$, (physics) and geometric, $\ell_{\mathrm{RUC}}$, 
(morphology) length-scales are related in a complicated manner. Moreover, rare event statistics can sometimes dictate the effective material response, such as in high cycle fatigue. Therefore, a rigorous RUC study should be conducted for any material of interest in order to establish microstructure-statistics-property relations (see Figure 1(b) for MSPR).

\section{Parallel Implementation and High Performance Computing}

During the past 20 years, development of large and fast HPC resources [230, 231] has been largely driven by the high-fidelity simulations in nuclear stockpile stewardship [232], weather and hurricane waves/surge predictions [233-236], space exploration [237, 238], biology [239-241], materials science [107, 108, 181, $242,243]$ and many others [244-247]. In the beginning, Moore's Law of processor's speed over CPU frequency [248] dominated gains in computational performance. Recently, the computational performance has increased through multi-level parallelism [231, 249, 250], while the per-CPU speed has remained nearly constant. For example, two of the world's fastest supercomputers in 2016 (Tianhe-2, National Supercomputer Center, China and Titan [251], Oak Ridge National Laboratory, USA) use co-processors and GPUs. Moreover, the next generation of supercomputers slated for 2017-2018 delivery as part of the DoE CORAL program will all be composed of heterogeneous architectures [252-254]. Therefore, in order to efficiently use these machines for scientific discovery, the scalability of numerical models (i.e., the ability to compute solutions faster in proportion to increased resources) will become ever more important [255].

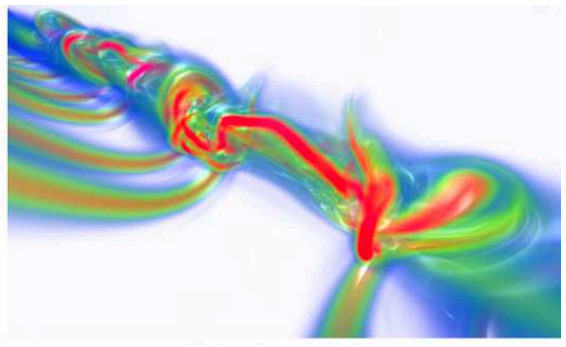

(a)

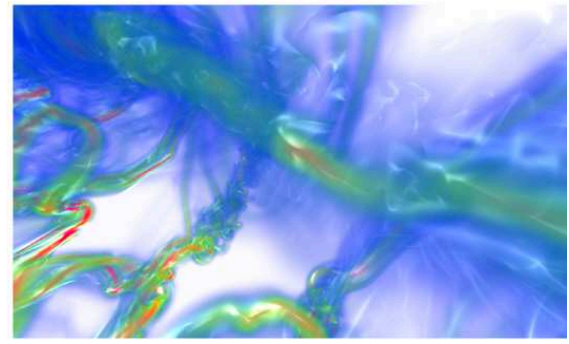

(b)

Figure 7: Counter-rotating vortices, initiation by ambient noise: details of a reconnection region. (a) $t / t_{0}=0.25$, (b) $t / t_{0}=$ 0.34. Reprinted from Comput. Methods in Appl. Mech. and Engrg., Vol. 197, Chatelain et al. Billion vortex particle direct numerical simulations of aircraft wakes, Pages No. 1296-1304, Copyright (2008), with permission from Elsevier, (see ref. [247].)

The expansion of numerical modeling tools for scientific simulations enabled Direct Numerical Modeling (DNM) (commonly called Direct Numerical Simulation (DNS) in the fluid mechanics community) of complex physical systems to flourish (see two reviews on DNS in fluid dynamics [256, 257]). For example, Chatelain et al. [247] presented the DNS of high Reynolds numbers aircraft wakes employing the vortex particle method [246] (see Figure 7). This particle method is based on the Navier-Stokes equations in vorticityvelocity form simulated using a Lagrangian discretization. Remeshing of the particles is employed in order to ensure the convergence.

Unfortunately, complex DNM/DNS simulations can require over one hundred billion computational cells to resolve complex physical phenomena such as turbulence, crack propagation, shear band formation, etc., especially for heterogeneous materials and mixtures [181]. Moreover, numerical simulations of PDEs suffer from limited scalability due to algorithmic complexity and parallel communication overhead [255, 258]. Therefore, even with recent increases in performance and availability of supercomputing environments, DNM/DNS often remains prohibitively expensive [259]. Fischer et al. [255] estimated that in order to take full advantage of the next-generation supercomputing platforms, the DNM/DNS would require approximately 
10 Trillion computational cells. Therefore, advanced numerical techniques that provide comparable accuracy to DNM/DNS are needed. Such numerical techniques would enable the effective use of the next generation of supercomputers, while also enabling simulation of larger domains with higher resolution on more accessible computing resources (i.e., commodity clusters).

Computational homogenization (see Section 4) is one of the methods that provides multi-level parallelism and reduces the computational cost, while maintaining high-fidelity representation of the physics (i.e., both microscale morphology and physical response when correctly specified at the microscale level). Due to the nested nature of the macro- and micro-problems, RUCs may be computed independently from each other, enabling efficient parallelization. Moreover, each RUC can also be simulated in parallel, leading to an efficient hierarchically parallel CH solver [107, 108]. This nested computational method, is often referred to as $\mathrm{FE}^{2}$, when two nested FE solutions are utilized or FE-FTT if a spectral solver is used at the RUC level with periodic boundary conditions.

(a)

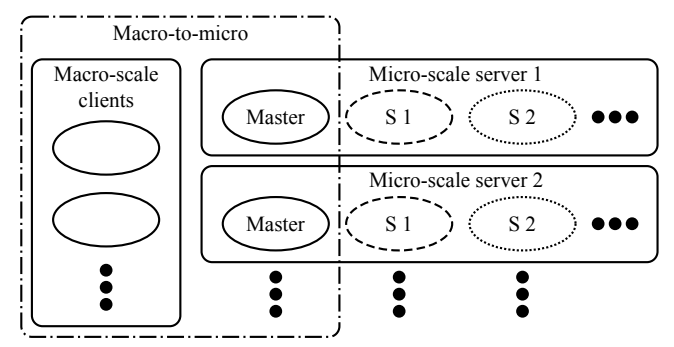

(b)

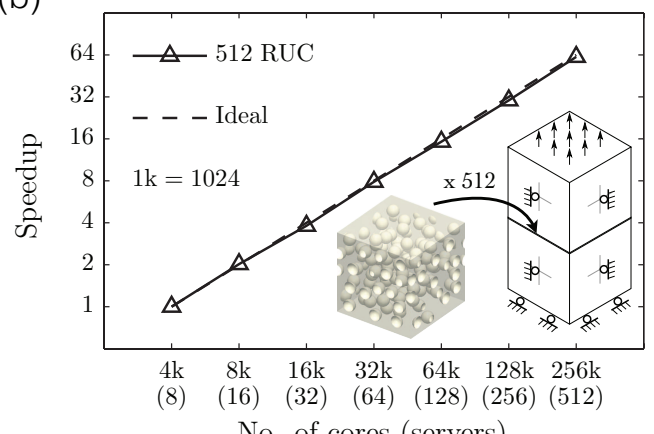

Figure 8: (a) Schematic of the hierarchically parallel communication framework. The communication between scales is performed only by the "master" processors of each server and the macro-scale client processors. Note that communication between servers is restricted to the equivalent task id (e.g., S1 on server 1 can communicate with S1 on server 2 , but not S2 on server 2). (b) Computational speedup of the hierarchically parallel multi-scale solver. The macro-scale is discretized with $17.5 \mathrm{k}$ finite elements and 9.8k nonlinear degrees of freedom (DOF), and is computed using 32 cores. Each of the 512 RUCs is discretized with $1.46 \mathrm{M}$ finite elements and $773 \mathrm{k}$ nonlinear DOFs, and is computed using 512 cores. The complete multi-scale simulation contains 747M finite elements and 396M DOFs. Figures taken from Mosby and Matouš [108].

There have been a few parallel implementations of the nested solution process [114, 183, 260-262], but they were not implemented in a true HPC setting that could take advantage of leadership class computing platforms and therefore showed only modest performance. Recently, Mosby and Matouš $[107,108]$ introduced a hierarchically parallel implementation of the $\mathrm{CH}$ formulation that enables large scale simulations. In their implementation, the macro-scale equilibrium is computed in parallel on the "client" processors, and the contributions from the individual RUCs are computed in parallel on the "servers" (see Fig. 8(a)). Furthermore, all communications are performed using dynamic point-to-point non-blocking messages that are overlaid with computations at both scales. Mosby and Matouš [108] performed a simulation consisting of 53.8 Billion finite elements with 28.1 Billion nonlinear equations that was solved on 393,216 computing cores (786,432 threads). Moreover, nearly ideal strong scalability has been achieved (see Figure 8(b)) from 4,096 to 262,144 computing cores on the Vulcan machine at LLNL. This exceptional performance holds excellent potential for deploying $\mathrm{CH}$ on future exascale computing platforms.

To demonstrate the ability of the $\mathrm{CH}$ solver to predict multiscale failure, we compute the response of a dual cantilever beam (DCB) using an isotropic viscous damage model at the microscale [181, 263]. Figure 9(a) shows the non-planar progressive failure of the interface governed by the microscale damage as well as crack tunneling. The extent of damage in the microscale at the marked points along the macroscale interface (point 1 and point 2) is displayed in Figure 9(b). The $\mathrm{CH}$ solver predicts that the fracture toughness of the interface is $G_{c} \in[141,175] \mathrm{J} / \mathrm{m}^{2}$ due to varying local strain rates. The mean toughness 

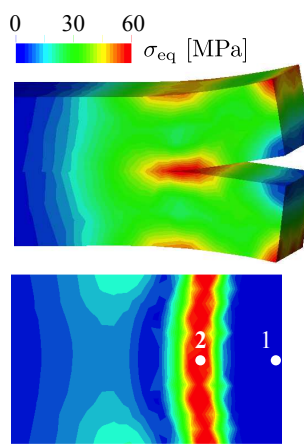

(a)
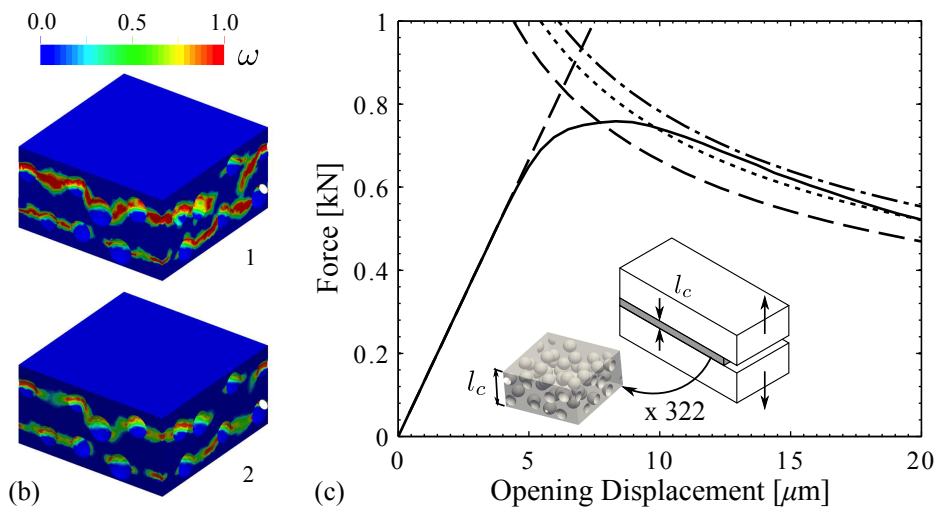

Figure 9: Fully coupled multiscale simulation of progressive mode-I failure of a DCB. The material cells are each discretized with $249 \mathrm{k}$ finite elements, and the total multiscale simulation contains $80 \mathrm{M}$ finite elements and $42.5 \mathrm{M}$ nonlinear DOFs. (a) The macroscale response. (b) Extent of damage in the microscale at the marked points in (a). (c) Multiscale force-displacement curves. Broken lines denote the LFM theory response for $G_{c}^{\min }=141 \mathrm{~J} / \mathrm{m}^{2}$ (dashed), $G_{c}^{\max }=175 \mathrm{~J} / \mathrm{m}^{2}$ (dash-dot), and $G_{c}^{\text {mean }}=162 \mathrm{~J} / \mathrm{m}^{2}$ (dotted). Figures taken from Mosby and Matouš [108].

of the interface is $G_{c}^{\text {mean }}=162 \mathrm{~J} / \mathrm{m}^{2}$. The critical interface first Piola-Kirchhoff traction is predicted as $t_{c}=\max \left\|\vec{p}_{M}\right\| \in[61,68.2] \mathrm{MPa}$, with $t_{c}^{\text {mean }}=65.6 \mathrm{MPa}$. Figure 9(c) shows that the multiscale response of the DCB, loaded in mode-I, is bounded by the Linear Fracture Mechanics (LFM) theory (broken lines). One can see that LFM theory over-predicts the maximum load by more than $20 \%\left(P_{\max }=759 \mathrm{~N}\right.$ from the $\mathrm{CH})$. The multiscale response is computed using up to $128 \mathrm{k}$ cores on the Mira supercomputer at Argonne National Laboratory.

\section{Reduced order Models, Data mining and Acceleration of Nonlinear Multiscale Methods}

As indicated in the previous section (Section 6), full resolution simulations are possible using HPC. However, for material design, optimization, and uncertainty quantification, HPC simulations are not always optimal due to their high computational cost. To lower this computational expense, the research community has focused on model reduction, as it can significantly reduce time and data storage requirements. Moreover, solution accelerators are essential to make progress (e.g. through improving initial guess) in nonlinear DNM/DNS and multiscale methods alike.

Although closely related, model reduction for single scale problems [264-267] differs to those in the multiscale setting [268-271]. In general, two nested BVPs are usually required in the multiscale setting. Considering the $\mathrm{CH}$ theory presented in Section 4, the macroscopic BVP provides boundary conditions for the microscale (process called localization), and the microscopic BVP yields the average flux (process called homogenization, i.e. the macroscopic stress tensor at a macroscale material point), and the macroscopic (homogenized) consistent tangent for implicit numerical schemes. Although the homogenization and localization processes are concatenated for the fully coupled $\mathrm{CH}$ implementation, these processes have distinctly different complexities when used in the context of model reduction. The localization requires solution of a nonlinear BVP (i.e. one of each RUC), whereas homogenization represents field averaging and solution of the macroscopic BVP (typically a smaller system of nonlinear equations).

Perhaps the simplest and efficient model reduction approach is macroscopic model calibration from detailed microscopic simulations. Note that this approach is only appropriate if the macroscopic model is known and accurate within the calibrated range. For example, Andrade and Tu [272] calibrated on the fly key material parameters of a phenomenological plasticity model at the macroscale from microscale computations using the discrete element method.

The Transformation Field Analysis (TFA) [206, 273], its nonuniform extension (NTFA) [274, 275], and eigenstrain based model reduction $[146,276]$ have been popular for small stain analyses in particular. Al- 
though quite appealing, the microstructure evolution is neglected by these approaches, which limits their predictive capabilities for certain nonlinear applications.

One popular model reduction technique is rooted in proper orthogonal decomposition (POD) [277]. Yvonnet and He [271] proposed a POD based model for both homogenization and localization of hyperelastic composites. Efficient integration of reduced-order equations in the POD context was performed by Hernández et al. [278]. Error bounding techniques for reduced order modeling using POD were proposed by Kerfriden et al. [279]. Other POD related reduction methods can be found in [280-285]. Although quite popular, POD is better suited for linear or weakly nonlinear processes (ordinary or partial differential equations), as it relies on a linear combination of a few basis vectors. Moreover, POD models can potentially drift from the true $\mathrm{CH}$ solution, since the method is directly coupled with the macroscale in terms of the reduced basis for most of the implementations.

Response Surface Models (RSM) [286] that substitute a microscale solution by an analytical function are also widely used. In this category, digital databases and/or discrete material maps are used to construct the overall homogenized macro-potentials or to directly homogenize the stress tensor [287-289]. In particular, Temizer and Wriggers [288] and Temizer and Zhodi [289] have applied a concept of virtual material experiments and developed discrete material maps. Here, a range of tri-axial stretches and rotations is applied to create a material homogenization map of the second Piola stress tensor. Another attempt to construct a RSM of the strain energy density function includes a technique based on the phase-space simplicial interpolation [290]. Unfortunately, the localized solution (i.e. detailed RUC solution) is usually lost using the RSMs.

Machine learning approaches like Neural Networks (NN) have also been employed to obtain approximate constitutive behavior in both single- and multi-scale settings. NN is inspired by the central nervous systems of animals, in particular the brain, and is used to estimate functions that depend on a large number of generally unknown inputs. NN has been used to approximate chemical potentials [291-293], constitutive behavior of concrete [267], geomaterials [294, 295], and has many other applications in mechanics of materials [296-300].

Recently, several dimension reduction techniques have been proposed that seek meaningful low-dimensional structures hidden in high-dimensional data [264, 266, 268, 301-307]. In the context of mechanics of materials, this high-dimensional data may represent full-field experimental measurements and/or detailed RUC simulations. Among these, Laplacian Eigenmaps [308] and Locally Linear Embeddings (LLE) [309] preserve only local geometry. Thus, these local methods can fail to capture the global pattern, especially when the data manifold contains a noisy signal $[304,310]$. Therefore, global techniques were developed to learn the underlying global geometry of a data set [303, 304, 311, 312]. Some global techniques, such as kernel principal component analysis (PCA) [312] and diffusion maps [311] are sensitive to the selection of kernels. On the other hand, a global technique like the complete isometric feature mapping (Isomap) approximately preserves relevant geometrical scales [303, 304, 307]. Isomap is rooted in classical Multidimensional Scaling (MDS), but attempts to preserve the intrinsic geometry of the data. Isomap has been used in several contexts. For example, a Gaussian process emulator of parametrized partial differential equations for single scale analysis has been developed by Xing et al. [264] and Triantafyllidis et al. [266]. Ganapathysubramanian and Zabaras employed Isomap for nonlinear dimension reduction of a data-driven stochastic input model [305], and Bhattacharjee and Matouš [268] used Isomap for manifold-based reduced order modeling of nonlinear heterogeneous hyperelastic materials as highlighted bellow.

Isomap is composed of three main steps. The first step involves identifying neighboring points on the manifold $\mathcal{M}$, which contains the set of data $\mathfrak{Z}$ associated with the corresponding set of input parameters $\mathfrak{T}$. The distance $D_{\mathfrak{T}}(i, j)$ is computed between pairs of points $i$ and $j$ in the input space $\mathfrak{T}$. Next, neighborhood relations are established using a graph. The manifold is represented by the graph, $G$, constructed from the points, $i$, using the $k$-rule, where $k$ nearest neighbors are connected through the graph edges with weight $D_{\mathfrak{T}}(i, j)$ (see Fig. 10(b)). The second step in Isomap is to approximate the geodesic distances $D_{M}(i, j)$ between all pairs of points on the manifold $\mathcal{M}$ by computing their shortest path distances $D_{G}(i, j)$ in the graph $G$. In the final step, Isomap involves the computation of an affinity matrix, $\bar{D}_{i j}=D_{G}^{2}(i, j)$, from the data set $\mathfrak{Z}$, and applies classical MDS techniques for constructing an embedding of the data in a $d$ dimensional Euclidean space $\mathcal{A}$. Thus, near and far points on $\mathcal{M}$ (by geodesic distance) are also near and far on $\mathcal{A}$ (by Euclidean distance) (see Fig. 10(c)). 
(a)

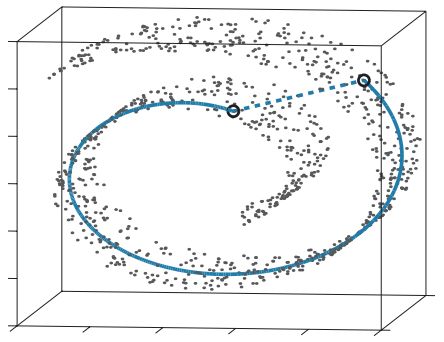

(b)

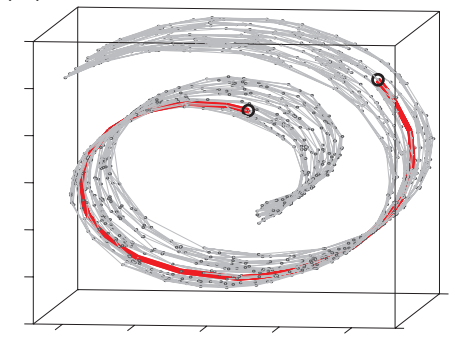

(c)

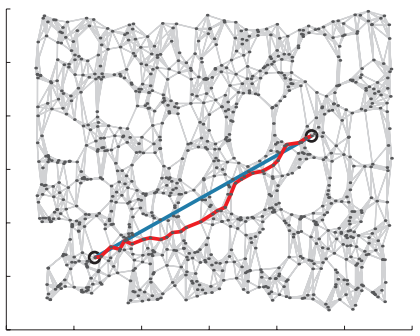

Figure 10: The "Swiss roll" data set (In mechanics of materials, this data can represent detailed RUC simulations and/or full-field in situ experimental measurements, for example), illustrating how Isomap exploits geodesic paths for nonlinear dimensionality reduction. (a) For two arbitrary points (circled) on a nonlinear manifold, their Euclidean distance in the highdimensional input space (length of dashed line in figure (a)) may not accurately reflect their intrinsic similarity, as measured by geodesic distance along the low-dimensional manifold (length of solid curve). (b) The neighbor-hood graph $G$ constructed in step one of Isomap (with $k=7$ and $N=1000$ data points) allows an approximation (red segments) of the true geodesic path to be computed efficiently in step two, as the shortest path in $G$. (c) The two-dimensional embedding recovered by Isomap in step three best preserves the shortest path distances in the neighborhood graph (overlaid). Straight lines in the embedding (blue) now represent simpler and cleaner approximations to the true geodesic paths than do the corresponding graph paths (red). Reprinted from Science, Vol. 290, Tenenbaum et al. A Global Geometric Framework for Nonlinear Dimensionality Reduction, Pages No. 2323-2326", Copyright (2000), with permission from AAAS, (see ref. [303].)

(a)

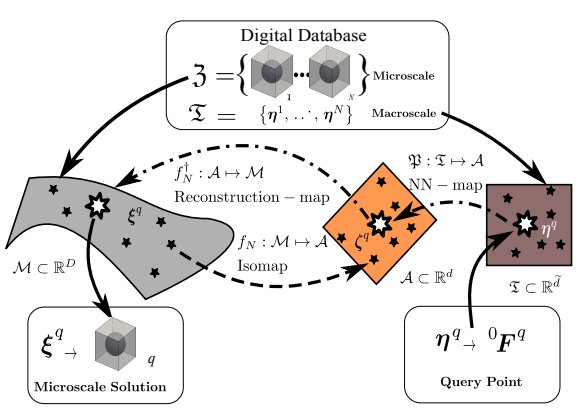

(b)

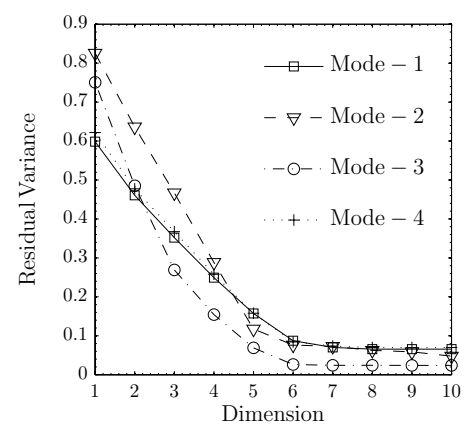

(c)

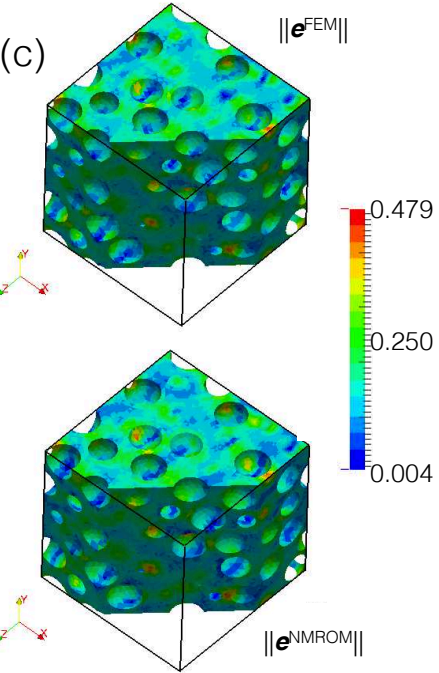

Figure 11: A manifold-based nonlinear reduced order model. (a) Schematic of the manifold-based nonlinear reduced order model and corresponding maps. (b) Isomap dimensionality. (c) Visualization of the microscopic effective Almansi strain $\left(\|\boldsymbol{e}\|_{\mathcal{F}}\right)$ (Note that particles are rigid-like and hence are removed for the purpose of visualization). Top: FEM simulation. Bottom: MNROM analysis. Figures taken from Bhattacharjee and Matouš [268]

To highlight the potential of a manifold-based nonlinear reduced order model (MNROM) in the context 
of $\mathrm{CH}$, we present the macro and microscale coupling approach from the nonlinear manifold point of view as developed in [268]. The MNROM is constructed from a digital database of $4 \times N=4 \times 4032$ detailed microscale nonlinear simulations of RUCs (denoted as $\mathfrak{Z}$ ) and the set of their associated macroscale loading parameters (denoted as $\mathfrak{T}$ ) (see Fig. 11(a)). Using the digital database, we build a high-dimensional manifold $\mathcal{M}$, and construct a low-dimensional Euclidean space $\mathcal{A}$. The inverse or reconstruction map, $f_{N}^{\dagger}: \mathcal{A} \mapsto \mathcal{M}$, that is employed in the localization phase (without additional BVP solutions over a RUC) is constructed in the sense of non-parametric regression. To close the MNROM, the low-dimensional parameters need to be linked to the physical macroscale loading conditions through the map $\mathfrak{P}$. This map is established using NN.

As an example, we investigate the behavior of randomly configured (statistically isotropic) particulate hyperelastic materials. A cubic RUC with side length $5 \mu \mathrm{m}$ contains 95 nonoverlapping spherical particles with $1 \mu \mathrm{m}$ diameter. The unit cell is loaded, as in the strain driven $\mathrm{CH}$, by the macroscopic deformation gradient, ${ }^{0} \boldsymbol{F}(\vec{X})={ }^{0} \boldsymbol{R}^{0} \boldsymbol{U}$. Recognizing that the microscopic response is invariant to the macroscopic rotation, we characterize macroscopic loading by three principal stretches, ${ }^{0} \lambda_{i}$, and three principal rotations, $\varphi^{i}$. The digital database is divided into four separate loading segments (referred to as Modes 1-4). After constructing the digital database, Isomap gives a reduced dimensionality of $d=6$ for each loading mode, down from the complete solution manifold with the dimension of $D=4,374,459$ (see Fig. 11(b)). Fig. 11(c) compares the microscopic effective Almansi strain within the matrix for both the FEM (top) and MNROM (bottom) analysis (localization step). As can be seen, the MNROM captures well the highly localized strain regions (see top cell surface and cross-sectional vertical surface) as well as the overall strain distribution (see the rest of the cell) without the need for additional solution of the BVP over the RUC. Note that this framework can also be used to accelerate fully coupled multiscale $\mathrm{CH}$ simulations (i.e. $\mathrm{FE}^{2}$ ). Developing MNROM for path dependent nonlinear material behavior is currently an active area of research.

\section{Verification, Validation and Uncertainty Quantification in Multiscale Computational Sci- ence and Engineering}

When evaluating the predictive ability of a simulation tool, systematic verification, validation, and uncertainty quantification is critical to understand the various sources of numerical and modeling errors. Verification is a process to ensure that the equations are being solved correctly by the numerical method, while validation denotes comparison of a simulation to experimental data to check physical validity of the governing equations. Verification and Validation $(\mathrm{V} \& \mathrm{~V})$ has first been formalized in the fluid mechanics community [313], and a book by Roache [229] discusses numerous techniques and case studies.

As numerical modeling strategies become increasingly complex and sophisticated, scientific software packages are being utilized for critical decision making in nuclear power reactors, weather, and hurricane wave surge modeling. Therefore, predictive abilities of simulation tools with Uncertainty Quantification (UQ) are critical.

The most complete definition of verification ensures both order of accuracy and consistency (solution approaches continuum form as discretization approaches zero) of the method [314]. Verification has traditionally been accomplished through comparing discretized solutions to analytical problems. However, analytical solutions for nonlinear problems only exist for simple scenarios. To overcome this limitation, Salari and Knupp used the method of manufactured solutions (MMS) [314]. This method rigorously tests many parts of a simulation code that affects the order of accuracy in the solution. MMS has been utilized extensively in the nonlinear solid mechanics community [315, 316]. A suite of verification problems for physics simulation codes used by Los Alamos National Laboratory (LANL), Sandia National Laboratories (SNL) and LLNL has played a fundamental role in the V\&V community [317]. A comprehensive discussion about $\mathrm{V} \& \mathrm{~V}$, its purpose and importance, as well as historical perspectives have been featured in many papers and monographs [229, 318-321].

Unfortunately, for multiscale modeling, there are only a limited number of verification studies, and even mathematical foundations for verification are lacking. For certain classes of problems in linear and nonlinear mechanics, Oden and coworkers [322] derived a posteriori error estimates and adaptivity measures for modeling error. This work has been applied to multiscale problems also [323, 324]. For general problems, 
Displacement loading, $\delta$

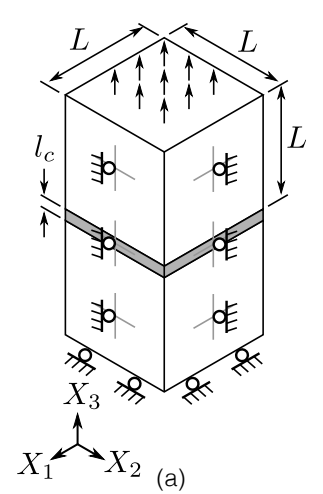

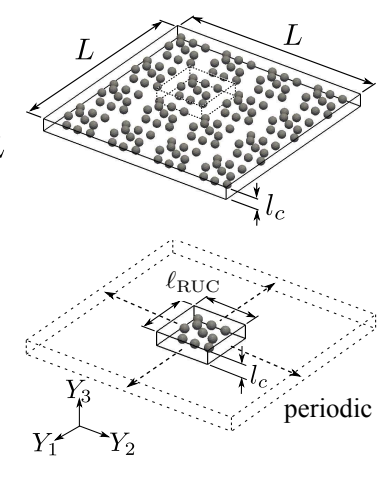

(b)

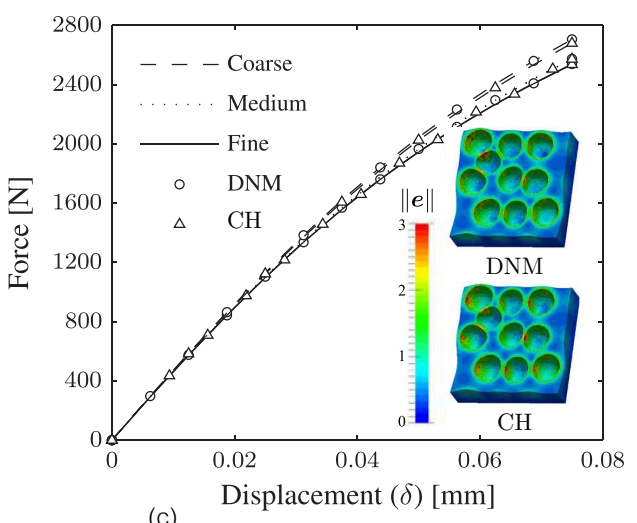

(c)

Figure 12: Verification of fully coupled CH for adhesive layers from Mosby and Matouš [107]. (a) Schematic illustrating macroscopic boundary conditions of adherends with $L=1 \mathrm{~mm}$ connected by a heterogeneous layer of thickness $l_{c}=0.0625 \mathrm{~mm}$. (b) The microscale heterogeneous layer for the DNM simulation (top) is based on a regular tessellation of the microstructure identified by the dotted box, and the cell used in the $\mathrm{CH}$ simulation with $\ell_{\mathrm{RUC}}=0.25 \mathrm{~mm}$ contains 10 spherical voids with $40 \mu \mathrm{m}$ diameter. Note that the $\mathrm{CH}$ simulation involves 16 computational cells, one for each macroscale cohesive element. (c) Comparison of force-displacement response between DNM and $\mathrm{CH}$ with inset comparing the microscale Almansi strain field.

one of the easiest verification approaches for $\mathrm{CH}$ is comparison to DNM at both the macro- and micro-scales. Mosby and Matouš [107] considered the constrained axial tension simulation (see Figure 12) using both DNM and $\mathrm{CH}$ for multi-scale cohesive modeling in a 3D finite strain setting. In this case, the microstructure of the heterogeneous adhesive layer is composed of monodisperse spherical voids, and the adhesive is modeled as hyperelastic. As the microscale mesh is refined, the solutions obtained from both DNM and CH at both macro- and micro-scales converge (see Figure 12(c)). While this is expected for simple loading cases where the macroscopic fields vary on a much larger length scale than heterogeneities, non-local effects $[34,325]$ can become apparent for irregular macroscopic geometries (and thus violating the separation of scales inequality (see Eq. 1)). Bishop et al. [326] illustrates this for a polycrystalline I-beam where the mean of the DNM solution deviates from a simple one-way coupled up-scaling scheme near sharp edges of the macroscopic domain. Once more, verification studies and procedures for multiscale modeling approaches are currently lacking.

As the complexity of the physical models coupled across several scales increases, the experimental and computational collaborations are becoming paramount. The close link between experimentalists and computational scientists is crucial for successful validation across all relevant scales and physical phenomena. In this direction, co-designed image-based modeling and experimental techniques can yield higher quality validation and provide insight into physical behavior often hidden from only a macroscopic perspective.

Similar to verification challenges, validation in the multiscale setting for complex material behavior is in the early stages. Obtaining high-resolution validation data across multiple scales is difficult [327-331]. Frequently, only two-dimensional microstructures are presented with qualitative description of the projected morphology and its evolution. Moreover, comparison to macroscopic measurement, e.g. macroscopic loading curve, is often the only extent of bulk validation.

To illustrate macroscale validation (upscaling), RVE/RUC simulations of a nickel-based superalloy from the work of Ghosh et al. [327] are presented. Careful reconstruction of the microstructure from FIB/SEM is considered, and two different microstructures are compared (with and without accounting for twinned crystals) in Figure 13(a). Note that the microstructure reconstructed with twins (preserving grain higher order correlations) better represents the experimental data. Figure 13(b-d) shows the micro-CT based macro- and micro-validation data of silica-reinforced polymer. Material is loaded in situ and imaged for quantitative assessment of void formation and growth. Moreover, the detailed macroscopic loading profile is obtained.

With the introduction and utilization of nondestructive three-dimensional imaging techniques in the materials science community [330,332], validation of microscale data is becoming feasible. Many papers 


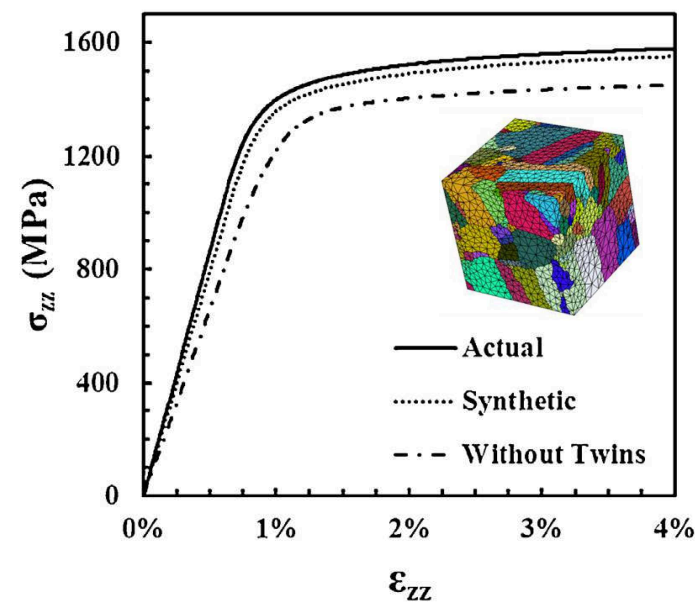

(a)

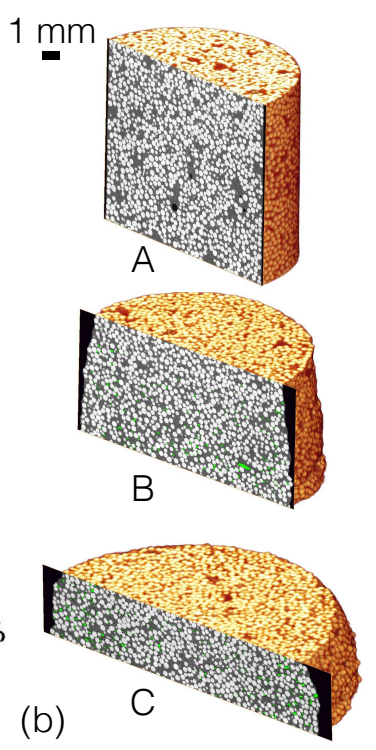

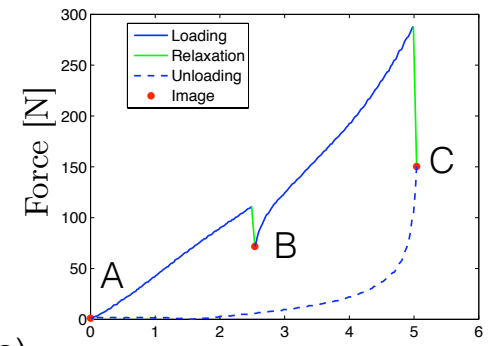

(c)

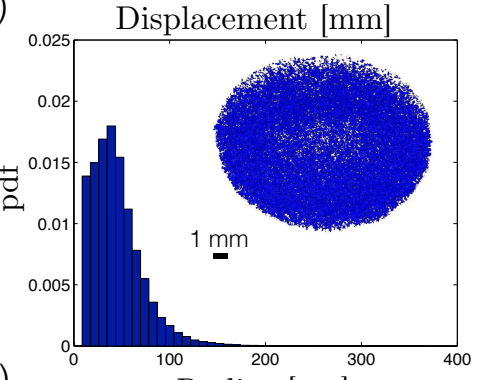

Figure 13: Validation examples for modeling of nonlinear materials. (a) Macro-scale validation of quasi-static loading for polycrystalline RUCs of nickel-based superalloys (microstructure in insert is that of nickel-based superalloys with (denoted synthetic) twins). Reprinted from Mechanics Research Communications, Ghosh et al. Multiscale modeling of polycrystalline nickel-based superalloys accounting for sub-grain microstructures, Copyright (2015), with permission from Elsevier, (see ref. [327]). (b-d) Multiscale validation experiment for silica-reinforced polymer with incremental microstructure imaging and in situ testing (from Computational Physics Group at the University of Notre Dame). (b) 3D reconstruction images of progressive debonding for points $\mathrm{A}, \mathrm{B}$ and $\mathrm{C}$ on the macroscopic loading curve in (c). Macroscale force-displacement curve indicating imaging points $\mathrm{A}, \mathrm{B}$ and $\mathrm{C}$ (red dots). (d) Void size distribution for loading point $\mathrm{C}$ with inset three-dimensional rendering of voids.

have utilized tomography for post-mortem or offline assessment [331, 333], and a few have performed in situ X-ray tomographic imaging during loading [330]. A powerful exploitation of tomographic approaches is threedimensional digital volume correlation $[334,335]$ where microscale changes are characterized over the loading history. Micro-scale in situ validation using tomographic approaches have been investigated for cellular materials (both metallic and polymeric) [330, 331, 336], metals [337], and woven composites [338, 339], just to name a few. Such image-based multiscale validation techniques with co-designed simulations will provide further confidence in physics simulation codes and advance the ICME paradigm (see Section 1).

A final yet nontrivial step in decision making abilities of predictive scientific codes is to understand and quantify uncertainties. Many of the simulation techniques including $\mathrm{CH}$ are deterministic, but uncertainties exist in the physical and experimental world that must be accounted for. Two broad classifications of uncertainty are recognized in the community: aleatory and epistemic uncertainty [313, 340]. Aleatory uncertainty refers to variability, irreducible uncertainty, inherent uncertainty, and stochastic uncertainty, whereas epistemic uncertainty denotes reducible uncertainty, subjective uncertainty, and model form uncertainty. When modeling heterogeneous materials, errors due to stochastic boundary conditions and material variability are sources of aleatory uncertainty. A common source of epistemic uncertainty in modeling heterogeneous materials relates to calibration of microscale constitutive models, where experimental data for calibrating all parameters of a microscale can be lacking.

In multiscale simulations, understanding and quantifying errors is critically important. How these errors initiate and propagate across scales is essential for determining the overall accuracy of any multiscale scheme. Moreover, this subsequently affects how the results from a multiscale scheme can be used. Multiscale simulations can efficiently transform epistemic uncertainty in parameters of coarse-grained models into the aleatoric uncertainty of the finer-grained models. Unfortunately, it is beyond the scope of this review article to go deeper into UQ at this point. Thus, we point an interested reader to a review article entitled "Uncertainty Quantification in Multiscale Simulation of Materials: A Prospective" [5] for more details on 
UQ in multiscale setting. More articles and books on general aspects of UQ can be found in [340-343].

\section{Conclusions and Future Directions}

Although existing computational science and engineering capabilities for modeling nonlinear material behavior are impressive, it is unclear how much impact they have had on materials engineering and design in general. Moreover, an integrated vision and/or framework that would be widely usable in multiscale computational materials engineering is lacking. Therefore, establishing microstructure-statistics-property relations underpinned by Integrated Computational Materials Engineering based on an image-based (datadriven) multiscale modeling framework with co-designed simulations and experiments has the potential to transform the materials field. Such a framework would ultimately lead to Virtual Materials Testing standards and aid in the development of new material formulations as well as decrease the time to market of innovative products.

In spite of the progress made over the past decades, a lot of work still remains to be done, and several technological barriers have to be mitigated to make integrated computational materials engineering a reality for a broad spectrum of materials applications. Among the ongoing challenges and expected trends the following ones are outlined:

- Fully coupled hierarchical and concurrent multiscale models in 3D

Without any doubt, three-dimensional multiscale simulations (both hierarchical and concurrent) that resolve all essential length (and time) scales are needed. Fully coupled multiscale simulations of realistic devices/components (test specimen) with complex physics are still uncommon and multiscale modeling is accomplished by the input and output of stand-alone codes mostly in twodimensions only. The progress to be made here is evidently coupled to the next item on Advances in Computing Capabilities.

- Advances in Computing Capabilities

Exponential growth in computer performance over the last several decades is one of the factors in integrated computational materials engineering feasibility. Moreover, high performance computing has had a dramatic effect on multiscale computational materials engineering. If current computer resources can do $X$, we envision that exascale machines will provide $\mathcal{O}(1000 X)$ improvement in terms of larger multiscale problems being solved, more physics being included, etc. Therefore, development and application of modern parallel computational methods will be paramount in the deployment of integrated computational materials engineering and utilization of the next generation of supercomputers (see Section 6).

- Intelligent Model Reduction - whether applied to computational or experimental data

Large 3D parallel simulations and experimental/imaging materials characterizations will necessitate the intelligent reduction of information required for the next level of materials integration. Mathematical/Statistical (e.g. machine learning) methods that seek meaningful low-dimensional structures hidden in high-dimensional multiscale data (both computational and experimental) will be important for a variety of tasks, i.e. multiscale solution accelerators, visualization, decision making, uncertainty quantification, reduced order model construction (see Section 7).

- Co-designed Simulations and Experiments in 3D

This review article has clearly illustrated the need for growing interaction with materials science. Materials science provides not only the required insight in the physical and mechanical behavior at small scales, but also the necessary quantitative data (parameters and models) on which any multiscale method relies. If the quality of this data is inadequate, not well understood or physical parameters are not reliable, then multiscale methods cannot possibly yield predictive 
results. Proper characterization of materials at small scales relies on strong collaboration between mechanics of materials experts with materials scientists. Cutting-edge research areas in 3D nondestructive experimental techniques with in situ measurements are not mature yet, but show a promising path for validation. Experimental measurements co-designed with simulations will have a profound impact on filling gaps in theoretical understanding and calibrating and validating computational methods. This can only be achieved by joining the expertise in experimental mechanics, computational mechanics and materials science.

- Verification, Validation and Uncertainty Quantification

Verification and Validation in computational science and engineering have been accepted as a key part of modern physics simulation codes and this trend must continue. Verification in a multiscale setting (i.e. how to efficiently construct verified solutions) is still an open area of work, and validation is linked to the discussion above on Co-designed Simulations and Experiments. Uncertainty is a complex issue with pervasive effects that deserves its own attention. Although UQ is a large and richly developed area of research in the context of computer modeling, applications of multiscale UQ techniques in materials science are still quite limited. Epistemic uncertainty in parameters of macroscopic models (i.e. global models) can be properly linked though multiscale simulations to aleatoric uncertainty of the microscopic (i.e. fine scale) models. Therefore, uncertainty quantification, propagation, mitigation and management, which are all relatively recent research areas in multiscale computational science and engineering, have to be further investigated (see Section 8).

- Multiscale versus Multi-level Description

Damage and fracture is one of the challenging problems that hierarchically cascades across all length scales, for example. Although multiscale modeling and multi-level materials design are related, these two endeavors are distinct. Multiscale techniques pertain to data and systems representing two or more distinct spatial and/or temporal scales whereas multi-level approaches aim at data and systems representing multiple levels of material arrangements. In the perspective of integrated computational materials engineering, multiscale modeling (both hierarchical and concurrent) is contributing to progress in multi-level materials design.

- Multiple Temporal Scales

A lot more attention has to be given to temporal scales and to spatial-temporal coupling. Many physical time scales in diffusion and small-scale deformation mechanisms cannot be accelerated. Likewise several small-scale processes happen very quickly, e.g. in many chemical reactions. Scale separation limitations and micro-inertia effects are also areas of interest. Therefore, capturing fully transient regimes and extreme spectra of temporal processes still remains a challenge.

- Cultural Barriers, Education and Workforce Readiness

If integrated computational materials engineering is to become part of the industrial design chain, a cultural change in the engineering and science disciplines is needed and will require the integration of computational science and engineering into curriculum at all levels (from baccalaureate to doctoral). Skills from statistics, mechanics, physics, chemistry, applied mathematics, scientific computing, data science, materials science, are all needed and will have to enrich traditional single-discipline curricula.

\section{Acknowledgments}

KM and AG were supported in part by the Department of Energy, National Nuclear Security Administration, under Award Number DE-NA0002377 as part of the Predictive Science Academic Alliance Program 
II. We would also like to acknowledge computational resources from the 2015 ASCR Leadership Computing Challenge (ALCC) under project number CSC188. KM would like to thank his former graduate research assistant, M. Mosby, for his numerous contributions to the multiscale modeling work presented in this review article.

\section{References}

[1] G. B. Olson, Computational design of hierarchically structured materials, Science 277 (1997) 1237-1242.

[2] D. McDowell, S. Ghosh, S. Kalidindi, Representation and computational structure-property relations of random media, JOM Journal of the Minerals, Metals and Materials Society 63 (2011) 45-51.

[3] N. R. Council, Integrated Computational Materials Engineering: A Transformational Discipline for Improved Competitiveness and National Security, The National Academies Press, Washington, DC, 2008.

[4] J. H. Panchal, S. R. Kalidindi, D. L. McDowell, Key computational modeling issues in integrated computational materials engineering, Computer-Aided Design 45 (2013) 4-25.

[5] A. Chernatynskiy, S. R. Phillpot, R. LeSar, Uncertainty quantification in multiscale simulation of materials: A prospective, Annual Review of Materials Research 43 (2013) 157-182.

[6] N. Science, T. Council, Materials Genome Initiative for Global Competitiveness, Technical Report, National Science and Technology Council, 2011. URL: http://www.whitehouse.gov/sites/default/files/microsites/ostp/materials_genome_initiative-final.pdf.

[7] E. Runge, E. Gross, Density-functional theory for time-dependent systems, Physical Review Letters 52 (1984) $997-1000$.

[8] A. D. Becke, Density-functional thermochemistry. III. The role of exact exchange, The Journal of chemical physics 98 (1993) 5648-5652.

[9] P. Geerlings, F. D. Proft, W. Langenaeker, Conceptual density functional theory, Chemical Reviews 103 (2003) $1793-$ 1873.

[10] J. Hafner, Ab-initio simulations of materials using VASP: Density-functional theory and beyond, Journal of Computational Chemistry 29 (2008) 2044-2078.

[11] A. Cohen, P. Mori-Sanchez, W. Yang, Insights into current limitations of density functional theory, Science 321 (2008) 792-794.

[12] K. Burke, L. Wagner, Dft in a nutshell, International Journal of Quantum Chemistry 113 (2013) 96.

[13] D. Porezag, T. Frauenheim, T. Kohler, Construction of tight-binding-like potentials on the basis of density functional theory - application to carbon, Physical Review B 51 (1995) 12947-12957.

[14] D. Hepburn, G. Ackland, Metallic-covalent interatomic potential for carbon in iron, Physical Review B 78 (2008) article 165115.

[15] J. Yasi, T. Noraget, D. Trinkle, Y. Qi, L. Hector, W. Curtin, Basal and prism dislocation cores in magnesium: comparison of first-principles and embedded-atom-potential methods predictions, Modelling and Simulation in Materials Science and Engineering 17 (2009) article 055012.

[16] L. Ventelon, F. Willaime, Generalized stacking-faults and screw-dislocation core-structure in bcc iron: A comparison between ab initio calculations and empirical potentials, Philosophical Magazine 90 (2010) 1063-1074.

[17] Z. Wu, M. Francis, W. Curtin, Magnesium interatomic potential for simulating plasticity and fracture phenomena, Modelling and Simulation in Materials Science and Engineering 23 (2015) article 015004.

[18] M. Finnis, Interatomic forces in condensed matter, Oxford University Press, 2003.

[19] I. M. Torrens, Interatomic potentials, Academic Press, New York, 1972.

[20] V. Vitek, Interatomic potentials for atomistic simulations, in: A. F. Voter (Ed.), MRS Bulletin, volume 21, Materials Research Society, 1996, p. 20.

[21] V. Yamakov, D. Wolf, S. Phillpot, A. Mukherjee, H. Gleiter, Dislocation processes in the deformation of nanocrystalline aluminium by molecular-dynamics simulation, Nature materials 1 (2002) 45-48.

[22] B. Holian, R. Ravelo, Fracture simulations using large scale molecular dynamics, Physical Review B 51 (1995) 1127511288.

[23] K. Kang, W. Cai, Size and temperature effects on the fracture mechanisms of silicon nanowires: Molecular dynamics simulations, International Journal of Plasticity 26 (2010) 1387-1401.

[24] A. Nair, E. Parker, P. Gaudreau, D. Farkas, R. Kriz, Size effects in indentation response of thin films at the nanoscale: A molecular dynamics study, International Journal of Plasticity 24 (2008) 2016-2031.

[25] F. Delogu, G. Cocco, Molecular dynamics investigation on the role of sliding interfaces and friction in the formation of amorphous phases, Physical Review B 71 (2005) 144108.

[26] D. Raabe, Computational Materials Science: the simulation of materials, microstructures and properties, Wiley-VCH, 1998.

[27] K. Kadau, T. C. Germann, P. S. Lomdahl, Molecular dynamics comes of age: 320 billion atom simulation on BlueGene/L, International Journal of Modern Physics C 17 (2006) 1755-1761.

[28] N. Metropolis, A. Rosenbluth, M. Rosenbluth, A. Teller, E. Teller, Equation of state calculations by fast computing machines, Journal of Chemical Physics 21 (1953) 1087.

[29] K. Binder, D. W. Heermann, Monte Carlo Simulation in Statistical Physics: An Introduction, volume 80 of Series in Solid-State Sciences, Springer, 1998.

[30] C. Truesdell, W. Noll, in: S. S. Antman (Ed.), The Non-Linear Field Theories of Mechanics, Springer Berlin Heidelberg, Berlin, Heidelberg, 2004, pp. 1-579. 
[31] R. Hill, Continuum micromechanics of elastoplastic polycrystals, Journal of the Mechanics and Physics of Solids 13 (1965) 89-101.

[32] A. Zaoui, Continuum micromechanics: Survey, Journal of Engineering Mechanics 128 (2002) 808-816.

[33] R. Hill, Elastic properties of reinforced solids: some theoretical principles, Journal of the Mechanics and Physics of Solids 11 (1963) 357-372.

[34] W. J. Drugan, J. R. Willis, A micromechanics-based nonlocal constitutive equation and estimates of representative volume element size for elastic composites, Journal of the Mechanics and Physics of Solids 44 (1996) 497-524.

[35] G. Povirk, Incorporation of microstructural information into models of two-phase materials, Acta metallurgica et materialia 43 (1995) 3199-3206.

[36] H. Kumar, C. Briant, W. Curtin, Using microstructure reconstruction to model mechanical behavior in complex microstructures, Mechanics of Materials 38 (2006) 818-832.

[37] N. Kumar, K. Matouš, P. Geubelle, Reconstruction of periodic unit cells of multimodal random particulate composites using genetic algorithms, Computational Materials Science 42 (2008) 352-367.

[38] H. Lee, M. Brandyberry, A. Tudor, K. Matouš, Three-dimensional reconstruction of statistically optimal unit cells of polydisperse particulate composites from microtomography, Physical Review E 80 (2009) 061301-1-061301-12.

[39] H. Moulinec, P. Suquet, A fast numerical method for computing the linear and nonlinear mechanical properties of composites, Comptes Rendus de l'Académie des Sciences II 318 (1994) 1417-1423.

[40] J. Michel, H. Moulinec, . P. Suquet, Effective properties of composite materials with periodic microstructure: a computational approach, Computer methods in applied mechanics and engineering 172 (1999) 109-143.

[41] M. Frigo, S. G. Johnson, The design and implementation of FFTW3, Proceedings of the IEEE 93 (2005) $216-231$.

[42] D. Pekurovsky, P3dfft: A framework for parallel computations of fourier transforms in three dimensions, SIAM Journal on Scientific Computing 34 (2012) C192-C209.

[43] Y. Guo, J. S. Curtis, Discrete element method simulations for complex granular flows, Annual Review of Fluid Mechanics 47 (2015) 21-46.

[44] E. B. Tadmor, M. Ortiz, R. Phillips, Mixed atomistic and continuum models of deformation in solids, Langmuir 12 (1996) 4529-4534.

[45] E. B. Tadmor, R. Phillips, M. Ortiz, Quasicontinuum analysis of defects in solids, Philosophical Magazine A73 (1996) $1529-1563$.

[46] W. E, B. Engquist, X. Lao, W. Ren, E. Vanden-Eijnden, Heterogeneous multiscale methods: A review, Communications in Computational Physics 2 (2007) 367-450.

[47] W. E, Principles of Multiscale Modeling, Cambridge University Press, 2011.

[48] J. Fish, Bridging the scales in nano engineering and science, Journal of Nanoparticle Research 8 (2006) 577-594.

[49] J. Fish (Ed.), Multiscale methods: bridging the scales in science and engineering, Oxford University Press, 2009.

[50] C. Miehe, C. Bayreuther, On multiscale FE analyses of heterogeneous structures: from homogenization to multigrid solvers, International Journal for Numerical Methods in Engineering 71 (2007) 1135-1180.

[51] J. Plews, C. Duarte, Bridging multiple structural scales with a generalized finite element method, International Journal for Numerical Methods in Engineering 102 (2014) 180-201.

[52] T. J. R. Hughes, G. R. Feijóo, L. Mazzei, J. Quincy, The variational multiscale method - a paradigm for computational mechanics, Computer Methods in Applied Mechanics and Engineering 166 (1998) 3-24.

[53] K. Garikipati, T. J. R. Hughes, A variational multiscale approach to strain localization - formulation for multidimensional problems, Computer Methods in Applied Mechanics and Engineering 188 (2000) 39-60.

[54] A. Abdulle, W. E, B. Engquist, E. Vanden-Eijnden, The heterogeneous multiscale method, Acta Numerica 21 (2012) $1-87$.

[55] K. Ridderbos, The coarse-graining approach to statistical mechanics: how blissful is our ignorance?, Studies In History and Philosophy of Science Part B: Studies In History and Philosophy of Modern Physics 33 (2002) 65-67.

[56] S. Ahuja, V. Yakhot, I. G. Kevrekidis, Computational coarse graining of a randomly forced one-dimensional Burgers equation, Physics of Fluids 20 (2008). Article 035111.

[57] I. Babuška, Homogenization and its application. mathematical and computational problems, in: Numerical solution of partial differential equations III, Academic Press, 1976, pp. 89-116.

[58] H. Öttinger, Beyond Equilibrium Thermodynamics, Wiley, 2005.

[59] M. Hütter, T. Tervoort, Finite anisotropic elasticity and material frame indifference from a nonequilibrium thermodynamics perspective, Journal of Non-Newtoninian Fluid Mechanics 152 (2008) 45-52.

[60] M. Hütter, T. Tervoort, Coarse graining in elasto-viscoplasticity: Bridging the gap from microscopic fluctuations to dissipation, Advances in Applied Mechanics 42 (2008) 253-317.

[61] M. Grmela, Why GENERIC?, Journal of Non-Newtonian Fluid Mechanics 165 (2010) 980-986.

[62] M. Grmela, Multiscale equilibrium and nonequilibrium thermodynamics in chemical engineering, Advances in chemical engineering 39 (2010) 75-129.

[63] G. Sachs, Zur ableitung einer fliessbedingung, Z. Ver. Dtsch. Ing. 72 (1928) 734-736.

[64] G. I. Taylor, Plastic strain in metals, Jnl. Inst. Met. 62 (1938) 307-324.

[65] J. D. Eshelby, The determination of the elastic field of an ellipsoidal inclusion, Proc. Royal Soc. London A241 (1957) 376-396.

[66] E. Kröner, Berechnung der elastischen konstanten des vielkristalls aus den konstanten des einkristalls, Z. Phys. 151 (1958) 504-518.

[67] Z. Hashin, S. Shtrikman, A variational approach to the theory of the elastic behaviour of multiphase materials, Journal of the Mechanics and Physics of Solids 11 (1963) 127-140. 
[68] T. Mori, K. Tanaka, Average stress in the matrix and average elastic energy of materials with misfitting inclusions, Acta Metallurgica 21 (1973) 571-574.

[69] I. Babuška, Solution of interface problems by homogenization - III, SIAM Journal on Mathematical Analysis 8 (1977) 923-937.

[70] J. R. Willis, Bounds on self-consistent estimates for the overall properties of anisotropic composites, Journal of the Mechanics and Physics of Solids 25 (1977) 185-202.

[71] E. Kröner, Zur plastischen verformung des vielkristalls, Acta Metallurgica 9 (1961) 155-161.

[72] J. W. Hutchinson, Bounds and self-consistent estimates for creep of polycrystalline metals, Proc. Royal Soc. London, Series A 394 (1976) 87-119.

[73] S. Nemat-Nasser, M. Obata, Rate-dependent finite elasto-plastic deformation of polycrystals, Proc. Royal Soc. London A407 (1986) 343-375.

[74] S. Nemat-Nasser, M. Hori, Micromechanics: overall properties of heterogeneous materials, Elsevier, Amsterdam, 1993.

[75] P. Ponte Castañeda, The effective mechanical properties of nonlinear isotropic composites, Journal of the Mechanics and Physics of Solids 39 (1991) 45-71.

[76] P. Suquet, Overall potentials and extremal surfaces of power law or ideally plastic materials, Journal of the Mechanics and Physics of Solids 41 (1993) 981-1002.

[77] P. Suquet, Continuum Micromechanics, volume 377 of CISM Courses and Lectures, Springer, 1997.

[78] J. R. Willis, Upper and lower bounds for nonlinear composite behaviour, Materials Science and Engineering A175 (1994) $7-14$.

[79] A. Zaoui, R. Masson, Micromechanics based modelling of plastic polycrystals: An affine formulation, Materials Science and Engineering A285 (2000) 418-424.

[80] A. L. Gurson, Continuum theory of ductile rupture by void nucleation and growth: Part I Yield criteria and flow rules for porous ductile media, J Eng Mater Technol Trans ASME 99 (1977) 2-15.

[81] J. Keller, A theorem on the conductivity of a composite medium, J. Math. Phys. 5 (1964) 548-549.

[82] J. Keller, Effective behavior of heterogeneous media, in: U. Landman (Ed.), Statistical Mechanics and Statistical Methods in Theory and Application: a tribute to Elliott W. Montroll, Plenum, 1977.

[83] A. Benssousan, J.-L. Lions, G. Papanicoulau, Asymptotic Analysis for Periodic Structures, North-Holland, AmsterdamNew York-Oxford, 1978.

[84] J.-L. Lions, Remarks on some asymptotic problems in composite materials and in perforated materials, in: S. NematNasser (Ed.), Variational Methods in Mechanics of Solids, Pergamon Press, 1979.

[85] E. Sanchez-Palencia, Non-homogeneous media and vibration theory, in: Lecture Notes in Physics, volume 127, SpringerVerlag, 1980.

[86] S. Nemat-Nasser, Retrospect and prospect. micromechanics, in: Proceedings of the 9th conference on Engineering Mechanics, College Station, TX, USA, 1992.

[87] M. Ortiz, Computational micromechanics, Computational Mechanics 18 (1996) 321-338.

[88] V. Tvergaard, Studies of the micromechanics of materials, European Journal of Mechanics A/Solids 16 (1997) 5-24.

[89] V. P. Smyshlyaev, N. A. Fleck, Bounds and estimates for linear composites with strain gradient effects, Journal of the Mechanics and Physics of Solids 42 (1994) 1851-1882.

[90] S. Forest, F. Pradel, K. Sab, Asymptotic analysis of heterogeneous Cosserat media, International Journal of Solids and Structures 38 (2001) 4585-4608.

[91] N. Triantafyllidis, S. Bardenhagen, The influence of scale size on the stability of periodic solids and the role of associated higher order gradient continuum models, Journal of the Mechanics and Physics of Solids 44 (1996) 1891-1928.

[92] V. P. Smyshlyaev, K. D. Cherednichenko, On rigorous derivation of strain gradient effects in the overall behaviour of periodic heterogeneous media, Journal of the Mechanics and Physics of Solids 48 (2000) 1325-1357.

[93] R. H. J. Peerlings, N. A. Fleck, Numerical analysis of strain gradient effects in periodic media, Journal de Physique IV 11 (2001) 153-160.

[94] P. Ponte Castañeda, New variational principles in plasticity and their application to composite materials, Journal of the Mechanics and Physics of Solids 40 (1992) 1757-1788.

[95] P. Ponte Castañeda, Second-order homogenization estimates for nonlinear composites incorporating field fluctuations: I theory, Journal of the Mechanics and Physics of Solids 50 (2002) 737-757.

[96] J. Fish, K. Shek, M. Pandheeradi, M. S. Shephard, Computational plasticity for composite structures based on mathematical homogenization: Theory and practice, Computer Methods in Applied Mechanics and Engineering 148 (1997) $53-73$.

[97] I. Doghri, C. Friebel, Effective elasto-plastic properties of inclusion-reinforced composites. study of shape, orientation and cyclic response., Mechanics of Materials 37 (2005) 45-68.

[98] I. Doghri, L. Brassart, L. Adam, J. Gerard, A second-moment incremental formulation for the mean-field homogenization of elasto-plastic composites, International Journal of Plasticity 27 (2011) 352-371.

[99] K. Matouš, P. H. Geubelle, Multiscale modelling of particle debonding in reinforced elastomers subjected to finite deformations, International Journal for Numerical Methods in Engineering 65 (2006) 190-223.

[100] P. Agoras, P. Ponte Castañeda, Homogenization estimates for multi-scale nonlinear composites, European Journal of Mechanics A/Solids 30 (2011) 828-843.

[101] P. Ponte Castañeda, E. Galipeau, Homogenization-based constitutive models for magnetorheological elastomers at finite strain, Journal of the Mechanics and Physics of Solids 59 (2011) 194-215.

[102] J. Fish, R. Fan, Mathematical homogenization of nonperiodic heterogeneous media subjected to large deformation transient loading, International Journal for Numerical Methods in Engineering 76 (2008) 1044-1064. 
[103] X. Markenscoff, C. Dascalu, Asymptotic homogenization analysis for damage amplification due to singular interaction of micro-cracks, Journal of the Mechanics and Physics of Solids 60 (2012) 1478-1485.

[104] Y. Yang, F. Ma, C. Lei, Y. Liu, J. Li, Nonlinear asymptotic homogenization and the effective behavior of layered thermoelectric composites, Journal of the Mechanics and Physics of Solids 61 (2013) 1768-1783.

[105] M. G. D. Geers, V. G. Kouznetsova, W. A. M. Brekelmans, Multi-scale computational homogenization: trends \& challenges, Journal of Computational and Applied Mathematics 234 (2010) 2175-2182.

[106] M. Geers, V. Kouznetsova, K. Matouš, J. Yvonnet, Homogenization methods and multiscale modeling: non-linear problems, in: E. Stein, R. de Borst, T. Hughes (Eds.), Encyclopedia of Computational Mechanics, John Wiley and Sons, NY, 2004

[107] M. Mosby, K. Matouš, Hierarchically parallel coupled finite strain multiscale solver for modeling heterogeneous layers, International Journal for Numerical Methods in Engineering 102 (2015) 748-765.

[108] M. Mosby, K. Matouš, Computational homogenization at extreme scales, Extreme Mechanics Letters 6 (2016) 68 - 74.

[109] P. M. Suquet, Local and global aspects in the mathematical theory of plasticity, in: A. Sawczuk, G. Bianchi (Eds.), Plasticity today: modelling, methods and applications, Elsevier Applied Science Publishers, London, 1985, pp. 279-310.

[110] S. Ghosh, K. Lee, S. Moorthy, Two scale analysis of heterogeneous elastic-plastic materials with asymptotic homogenization and Voronoi cell finite element model, Computer Methods in Applied Mechanics and Engineering 132 (1996) $63-116$.

[111] R. J. M. Smit, W. A. M. Brekelmans, H. E. H. Meijer, Prediction of the mechanical behaviour of non-linear systems by multi-level finite element modeling, Computer Methods in Applied Mechanics and Engineering 155 (1998) $181-192$.

[112] C. Miehe, J. Schröder, J. Schotte, Computational homogenization analysis in finite plasticity. Simulation of texture development in polycrystalline materials, Computer Methods in Applied Mechanics and Engineering 171 (1999) 387418.

[113] C. Miehe, J. Schotte, J. Schröder, Computational micro-macro transitions and overall moduli in the analysis of polycrystals at large strains, Computational Materials Science 16 (1999) 372-382.

[114] F. Feyel, J. L. Chaboche, $\mathrm{FE}^{2}$ multiscale approach for modelling the elasto-viscoplastic behaviour of long fibre $\mathrm{SiC} / \mathrm{Ti}$ composite materials, Computer Methods in Applied Mechanics and Engineering. 183 (2000) 309-330.

[115] K. Terada, M. Hori, T. Kyoya, N. Kikuchi, Simulation of the multi-scale convergence in computational homogenization approaches, International Journal of Solids and Structures 37 (2000) 2285-2311.

[116] S. Ghosh, K. Lee, P. Raghavan, A multi-level computational model for multi-scale damage analysis in composite and porous materials, International Journal of Solids and Structures 38 (2001) 2335-2385.

[117] V. G. Kouznetsova, W. A. M. Brekelmans, F. P. T. Baaijens, An approach to micro-macro modeling of heterogeneous materials, Computational Mechanics 27 (2001) 37-48.

[118] K. Terada, N. Kikuchi, A class of general algorithms for multi-scale analyses of heterogeneous media, Computer Methods in Applied Mechanics and Engineering 190 (2001) 5247-5464.

[119] C. Miehe, J. Schröder, M. Becker, Computational homogenization analysis in finite elasticity: material and structural instabilities on the micro- and macro-scales of periodic composites and their interaction, Computer Methods in Applied Mechanics and Engineering 191 (2002) 4971-5005.

[120] K. Matouš, M. G. Kulkarni, P. H. Geubelle, Multiscale cohesive failure modeling of heterogeneous adhesives, Journal of the Mechanics and Physics of Solids 56 (2008) 1511-1533.

[121] O. van der Sluis, P. J. G. Schreurs, W. A. M. Brekelmans, H. E. H. Meijer, Overall behaviour of heterogeneous elastoviscoplastic materials: effect of microstructural modelling, Mechanics of Materials 32 (2000) 449-462.

[122] C. Miehe, Strain-driven homogenization of inelastic microstructures and composites based on an incremental variational formulation, International Journal for Numerical Methods in Engineering 55 (2002).

[123] T. Kanit, S. Forest, I. Galliet, V. Mounoury, D. Jeulin, Determination of the size of the representative volume element for random composites: statistical and numerical approach, International Journal of Solids Structures 40 (2003) $3647-3679$.

[124] T. Kanit, F. N'Guyen, S. Forest, D. Jeulin, M. Reed, S. Singleton, Apparent and effective physical properties of heterogeneous materials: Representativity of samples of two materials from food industry, Computer Methods in Applied Mechanics and Engineering 195 (2006) 3960-3982.

[125] Z. Khisaeva, M. Ostoja-Starzewski, On the size of RVE in finite elasticity of random composites, Journal of Elasticity 85 (2006) 153173.

[126] D. Perić, E. de Souza Neto, R. Feijóo, M. Partovi, A. Molina, On micro-to-macro transitions for multi-scale analysis of non-linear heterogeneous materials: unified variational basis and finite element implementation, International Journal for Numerical Methods in Engineering 87 (2011) 149-170.

[127] S. D. Mesarovic, J. Padbidri, Minimal kinematic boundary conditions for simulations of disordered microstructures, Philosophical Magazine 85 (2005) 65-76.

[128] H. M. Inglis, P. H. Geubelle, K. Matouš, Boundary condition effects on multiscale analysis of damage localization, Philosophical Magazine 88 (2008) 2373-2397.

[129] F. Larsson, K. Runesson, S. Saroukhani, R. Vafadari, Computational homogenization based on a weak format of microperiodicity for RVE-problems, Computer Methods in Applied Mechanics and Engineering 200 (2011) 11-26.

[130] E. W. C. Coenen, V. G. Kouznetsova, M. G. D. Geers, Novel boundary conditions for strain localization analyses in microstructural volume elements, International Journal for Numerical Methods in Engineering 90 (2012) 1-21.

[131] I. Özdemir, W. A. M. Brekelmans, M. G. D. Geers, Computational homogenization for heat conduction in heterogeneous solids, International Journal for Numerical Methods in Engineering 73 (2008) 185-204.

[132] I. Özdemir, W. A. M. Brekelmans, M. G. D. Geers, FE ${ }^{2}$ computational homogenization for the thermo-mechanical analysis of heterogeneous solids, Computer Methods in Applied Mechanics and Engineering 198 (2008) 602-613. 
[133] F. Souza, D. Allen, Y.-R. Kim, Multiscale model for predicting damage evolution in composites due to impact loading, Composites Science and Technology 68 (2008) 2624-2634.

[134] K. Pham, V. Kouznetsova, M. Geers, Transient computational homogenization for heterogeneous materials under dynamic excitation, Journal of the Mechanics and Physics of Solids 61 (2013) 2125-2146.

[135] A. Sridhar, V. Kouznetsova, M. Geers, Homogenization of locally resonant acoustic metamaterials towards an emergent enriched continuum, Computational Mechanics 57 (2016) 423-435.

[136] C. Miehe, J. Schröder, J. Schotte, Computational homogenization analysis in finite plasticity. Simulation of texture development in polycrystalline materials, Computer Methods in Applied Mechanics and Engineering 171 (1999) 387418.

[137] C. Miehe, A. Koch, Computational micro-to-macro transition of discretized microstructures undergoing small strain, Archives in Applied Mechanics 72 (2002) 300-317.

[138] F. Larsson, K. Runesson, F. Su, Variationally consistent computational homogenization of transient heat flow, International Journal for Numerical Methods in Engineering 81 (2010) 1659-1686.

[139] A. Javili, G. Chatzigeorgiou, P. Steinmann, Computational homogenization in magneto-mechanics, International Journal of Solids and Structures 50 (2013) 4197-4216.

[140] D. Zäh, C. Miehe, Computational homogenization in dissipative electro-mechanics of functional materials, Computer Methods in Applied Mechanics and Engineering. 267 (2013) 487-510.

[141] I. Niyonzima, R. Sabariego, P. Dular, C. Geuzaine, Nonlinear computational homogenization method for the evaluation of eddy currents in soft magnetic composites, IEEE Transactions on Magnetics 50 (2014) 61-64.

[142] M. Keip, P. Steinmann, J. Schröder, Two-scale computational homogenization of electro-elasticity at finite strains, Computer Methods in Applied Mechanics and Engineering 278 (2014) 62-79.

[143] C. Miehe, D. Vallicotti, S. Teichtmeister, Homogenization and multiscale stability analysis in finite magneto-electroelasticity. Application to soft matter EE, ME and MEE composites, Computer Methods in Applied Mechanics and Engineering 300 (2016) 294-346.

[144] F. Nilenius, F. Larsson, K. Lundgren, K. Runesson, Computational homogenization of diffusion in three-phase mesoscale concrete, Computational Mechanics 54 (2014) 461-472.

[145] M. Ohman, F. Larsson, K. Runesson, Computational homogenization of liquid-phase sintering with seamless transition from macroscopic compressibility to incompressibility, Computer Methods in Applied Mechanics and Engineering 266 (2013) 219-228.

[146] Z. Yuan, T. Jiang, J. Fish, G. Morscher, Reduced-order multiscale-multiphysics model for heterogeneous materials, International Journal for Multiscale Computational Engineering 12 (2014) 45-64.

[147] F. Su, F. Larsson, K. Runesson, Computational homogenization of coupled consolidation problems in micro-heterogeneous porous media, International Journal for Numerical Methods in Engineering 88 (2011) 1198-1218.

[148] X. Zhuang, Q. Wang, H. Zhu, A 3D computational homogenization model for porous material and parameters identification, Computational Materials Science 96 (2015) 536-548.

[149] R. Jänicke, B. Quintal, H. Steeb, Numerical homogenization of mesoscopic loss in poroelastic media, European Journal of Mechanics - A/Solids 49 (2015) 382-395.

[150] K. Gao, J. van Dommelen, P. Gäransson, M. Geers, A homogenization approach for characterization of the fluid-solid coupling parameters in biot's equations for acoustic poroelastic materials, Journal of Sound and Vibration 351 (2015) 251-267.

[151] V. Nguyen, L. Noels, Computational homogenization of cellular materials, International Journal of Solids and Structures 51 (2014) 2183-2203.

[152] A. Iltchev, V. Marcadon, S. Kruch, S. Forest, Computational homogenisation of periodic cellular materials: Application to structural modelling, International Journal of Mechanical Sciences 93 (2015) 240-255.

[153] J. Segurado, J. Llorca, Simulation of the deformation of polycrystalline nanostructured ti by computational homogenization, Computational Materials Science 76 (2013) 3-11.

[154] K. Matouš, A. M. Maniatty, Multiscale modeling of elasto-viscoplastic polycrystals subjected to finite deformations, Interaction and multiscale mechanics 2 (2009) 375-396.

[155] S. Fillep, J. Mergheim, P. Steinmann, Computational homogenization of rope-like technical textiles, Computational Mechanics 55 (2015) 577-590.

[156] X. Liu, Y. Liang, Q. Duan, B. Schrefler, Y. Du, A mixed finite element procedure of gradient cosserat continuum for second-order computational homogenisation of granular materials, Computational Mechanics 54 (2014) 1331-1356.

[157] M. Wierszycki, K. Szajek, T. Lodygowski, M. Nowak, A two-scale approach for trabecular bone microstructure modeling based on computational homogenization procedure, Computational Mechanics 54 (2014) 287-298.

[158] R. Breuls, B. Sengers, C. Oomens, C. Bouten, F. Baaijens, Predicting local cell deformations in engineered tissue constructs: a multilevel finite element approach, ASME Journal of Biomechanical Engineering 124 (2002) 198-207.

[159] A. Salvadori, E. Bosco, D. Grazioli, A computational homogenization approach for Li-ion battery cells: Part 1formulation, Journal of the Mechanics and Physics of Solids 65 (2014) 114-137.

[160] V. G. Kouznetsova, M. G. D. Geers, W. A. M. Brekelmans, Advanced constitutive modeling of heterogeneous materials with a gradient-enhanced computational homogenization scheme, International Journal for Numerical Methods in Engineering 54 (2002) 1235-1260.

[161] M. Geers, V. Kouznetsova, W. Brekelmans, Multi-scale second-order computational homogenization of microstructures towards continua, International Journal for Multiscale Computational Engineering 1 (2003) 371-386.

[162] V. Kouznetsova, M. Geers, W. Brekelmans, Size of a representative volume element in a second-order computational homogenization framework, International Journal for Multiscale Computational Engineering 2 (2004) 575-598. 
[163] V. Kouznetsova, M. Geers, W. Brekelmans, Multi-scale second-order computational homogenization of multi-phase materials: a nested finite element solution strategy, Computer Methods in Applied Mechanics and Engineering 193 (2004) 5525-5550.

[164] L. Kaczmarczyk, C. Pearce, N. Bicanic, Scale transition and enforcement of RVE boundary conditions in second-order computational homogenization, International Journal for Numerical Methods in Engineering 74 (2008) 506-522.

[165] L. Kaczmarczyk, C. Pearce, N. Bicanic, Studies of microstructural size effect and higher-order deformation in second-order computational homogenization, Computers \& Structures 88 (2010) 1383-1390.

[166] A. Bacigalupo, L. Gambarotta, Nonlocal computational homogenization of periodic masonry, International Journal for Multiscale Computational Engineering 9 (2011) 565-587.

[167] J. Fish, S. Kuznetsov, Computational continua, International Journal for Numerical Methods in Engineering 84 (2010) 774-802.

[168] J. Fish, V. Filonova, D. Fafalis, Computational continua revisited, International Journal for Numerical Methods in Engineering 102 (2015) 332-378.

[169] S. Loehnert, T. Belytschko, A multiscale projection method for macro/microcrack simulations, International Journal for Numerical Methods in Engineering 71 (2007) 1466-1482.

[170] T. Belytschko, S. Loehnert, J. H. Song, Multiscale aggregating discontinuities: A method for circumventing loss of material stability, International Journal for Numerical Methods in Engineering 73 (2008) 869-894.

[171] T. Hettich, A. Hund, E. Ramm, Modeling of failure in composites by X-FEM and level sets within a multiscale framework, Computer Methods in Applied Mechanics and Engineering 197 (2008) 414-424.

[172] T. Massart, R. Peerlings, M. Geers, An enhanced multi-scale approach for masonry wall computations with localization of damage, International Journal for Numerical Methods in Engineering 69 (2007) 1022-1059.

[173] T. Massart, R. Peerlings, M. Geers, Structural damage analysis of masonry walls using computationa homogenization, International Journal of Damage Mecanics 16 (2007) 199-226.

[174] E. Coenen, V. Kouznetsova, M. Geers, Multi-scale continuous-discontinuous framework for computationalhomogenization-localization, Journal of the Mechanics and Physics of Solids 60 (2012) 1486-1507.

[175] E. Coenen, V. Kouznetsova, E. Bosco, M. Geers, A multi-scale approach to bridge microscale damage and macroscale failure: a nested computational homogenization-localization framework, International Journal of Fracture 178 (2012) $157-178$.

[176] E. Bosco, V. Kouznetsova, E. Coenen, M. G. D. Geers, A. Salvadori, A multiscale framework for localizing microstructures towards the onset of macroscopic discontinuity, Computational Mechanics 54 (2014) 299-319.

[177] E. Bosco, V. Kouznetsova, M. Geers, Multi-scale computational homogenization-localization for propagating discontinuities using X-FEM, International Journal for Numerical Methods in Engineering 102 (2015) 496-527.

[178] M. Geers, E. Coenen, V. Kouznetsova, Multi-scale computational homogenization of structured thin sheets, Modelling and Simulation in Materials Science and Engineering 15 (2007) S393-S404.

[179] E. W. C. Coenen, V. G. Kouznetsova, M. G. D. Geers, Computational homogeneization for heterogeneous thin sheets, International Journal for Numerical Methods in Engineering 83 (2010) 1180-1205.

[180] C. Helfen, S. Diebels, Computational homogenisation of composite plates: Consideration of the thickness change with a modified projection strategy, Computers \& Mathematics with Applications 67 (2014) 1116-1129.

[181] M. Mosby, K. Matouš, On mechanics and material length scales of failure in heterogeneous interfaces using a finite strain high performance solver, Modelling and Simulation in Materials Science and Engineering 23 (2015) 085014.

[182] C. Verhoosel, J. Remmers, M. Gutierrez, R. de Borst, Computational homogenization for adhesive and cohesive failure in quasi-brittle solids, International Journal for Numerical Methods in Engineering 83 (2010) 1155-1179.

[183] V. Nguyen, O. Lloberas-Valls, M. Stroeven, L. Sluys, Computational homogenization for multiscale crack modeling. implementational and computational aspects, International Journal for Numerical Methods in Engineering 89 (2012) $192-226$.

[184] C. Hirschberger, S. Ricker, P. Steinmann, N. Sukumar, Computational multiscale modelling of heterogeneous material layers, Engineering Fracture Mechanics 76 (2009) 793-812.

[185] L. De Lorenzis, P. Wriggers, Computational homogenization of rubber friction on rough rigid surfaces, Computational Materials Science 77 (2013) 264-280.

[186] I. Temizer, Multiscale thermomechanical contact: Computational homogenization with isogeometric analysis, International Journal for Numerical Methods in Engineering 97 (2014) 582-607.

[187] I. Temizer, Computational homogenization of soft matter friction: Isogeometric framework and elastic boundary layers, International Journal for Numerical Methods in Engineering 100 (2014) 953-981.

[188] H. Lee, A. S. Gillman, K. Matouš, Computing overall elastic constants of polydisperse particulate composites from microtomographic data, Journal of the Mechanics and Physics of Solids 59 (2011) 1838-1857.

[189] A. Gillman, K. Matouš, S. Atkinson, Microstructure-statistics-property relations of anisotropic polydisperse particulate composites using tomography, Physical Review E 87 (2013) 022208-1-022208-19.

[190] C. Shuck, M. Frazee, A. Gillman, M. Beason, I. Gunduz, K. Matouš, R. Winarski, A. Mukasyan, X-ray nanotomography and focused ion beam sectioning for quantitative three-dimensional analysis of nanocomposites, Journal of Synchrotron Radiation 23 (2016) 990-996.

[191] R. P. Winarski, M. V. Holt, V. Rose, P. Fuesz, D. Carbaugh, C. Benson, D. Shu, D. Kline, G. B. Stephenson, I. McNulty, et al., A hard X-ray nanoprobe beamline for nanoscale microscopy, Journal of Synchrotron Radiation 19 (2012) 1056-1060.

[192] S. Gallier, F. Hiernard, Microstructure of composite propellants using simulated packings and x-ray tomography, Journal of propulsion and power 24 (2008) 154-157.

[193] B. Collins, F. Maggi, K. Matouš, T. Jackson, J. Buckmaster, Using tomography to characterize heterogeneous propellants, 
in: 46th AIAA Aerospace Sciences Meeting and Exhibit, American Institute of Aeronautics and Astronautics, 2008.

[194] T. Aste, M. Saadatfar, T. Senden, Geometrical structure of disordered sphere packings, Physical Review E 71 (2005) 061302.

[195] S. Rolland du Roscoat, M. Decain, C. Geindreau, X. Thibault, J.-F. Bloch, et al., Microstructural analysis of paper using synchrotron X-ray microtomography: numerical estimation of the permeability and effective thermal conductivity, Appita Journal: Journal of the Technical Association of the Australian and New Zealand Pulp and Paper Industry 61 (2008) 286.

[196] D. Bouvard, J.-M. Chaix, R. Dendievel, A. Fazekas, J. Létang, G. Peix, D. Quenard, Characterization and simulation of microstructure and properties of EPS lightweight concrete, Cement and Concrete Research 37 (2007) 1666-1673.

[197] G. Möbus, B. J. Inkson, Nanoscale tomography in materials science, Materials Today 10 (2007) 18-25.

[198] M. Groeber, B. Haley, M. Uchic, D. Dimiduk, S. Ghosh, 3D reconstruction and characterization of polycrystalline microstructures using a FIB-SEM system, Materials Characterization 57 (2006) 259-273.

[199] M. D. Uchic, M. A. Groeber, D. M. Dimiduk, J. Simmons, 3D microstructural characterization of nickel superalloys via serial-sectioning using a dual beam FIB-SEM, Scripta Materialia 55 (2006) 23-28.

[200] J. R. Wilson, J. S. Cronin, S. A. Barnett, S. J. Harris, Measurement of three-dimensional microstructure in a LiCoO 2 positive electrode, Journal of Power Sources 196 (2011) 3443-3447.

[201] A. Schwartz, M. Kumar, B. Adams, D. Field, Electron Backscatter Diffraction in Materials Science, Routledge research global environmental change series, Springer US, 2000. URL: https://books.google.com/books?id=TOEhb-Nqe04C.

[202] C. Taylor, C. Figueroa, Patient-specific modeling of cardiovascular mechanics, Annual Review of Biomedical Engineering 11 (2009) 109-134. PMID: 19400706.

[203] A. Gillman, G. Amadio, K. Matouš, T. Jackson, Third-order thermo-mechanical properties for packs of Platonic solids using statistical micromechanics, Proceedings of the Royal Society of London A: Mathematical, Physical and Engineering Sciences 471 (2015) 20150060-1-20150060-20.

[204] A. Gillman, K. Matouš, Third-order model of thermal conductivity for random polydisperse particulate materials using well-resolved statistical descriptions from tomography, Physics Letters A 378 (2014) 3070-3073.

[205] J. Segurado, J. Llorca, A numerical approximation to the elastic properties of sphere-reinforced composites, Journal of the Mechanics and Physics of Solids 50 (2002) 2107-2121.

[206] K. Matouš, Damage evolution in particulate composite materials, International Journal of Solids and Structures 40 (2003) $1489-1503$

[207] M. G. Kulkarni, P. H. Geubelle, K. Matouš, Multi-scale modeling of heterogeneous adhesives: Effect of particle decohesion, Mechanics of Materials 41 (2009) 573-583.

[208] J. Yvonnet, Q.-C. He, C. Toulemonde, Numerical modelling of the effective conductivities of composites with arbitrarily shaped inclusions and highly conducting interface, Composites Science and Technology 68 (2008) 2818-2825.

[209] R. Largenton, J.-C. Michel, P. Suquet, Extension of the nonuniform transformation field analysis to linear viscoelastic composites in the presence of aging and swelling, Mechanics of Materials 73 (2014) 76-100.

[210] J. Segurado, C. Gonzalez, J. Llorca, A numerical investigation of the effect of particle clustering on the mechanical properties of composites, Acta Materialia 51 (2003) 2355-2369.

[211] J. Zeman, M. Šejnoha, From random microstructures to representative volume elements, Modelling and Simulation in Materials Science and Engineering 15 (2007) S325-S335.

[212] S. Torquato, Random Heterogeneous Materials: Microstructure and Macroscopic Properties, volume 16, Springer Science \& Business Media, 2002

[213] C. Yeong, S. Torquato, Reconstructing random media, Physical Review E 57 (1998) 495-506.

[214] B. Bochenek, R. Pyrz, Reconstruction of random microstructures-a stochastic optimization problem, Computational Materials Science 31 (2004) 93-112.

[215] B. Collins, K. Matouš, D. Rypl, Three-dimensional reconstruction of statistically optimal unit cells of multimodal particulate composites, International Journal for Multiscale Computational Engineering 8 (2010) 489-507.

[216] Y. Jiao, F. Stillinger, S. Torquato, Modeling heterogeneous materials via two-point correlation functions. II. algorithmic details and applications, Physical Review E 77 (2008) 031135.

[217] D. T. Fullwood, S. R. Niezgoda, S. R. Kalidindi, Microstructure reconstructions from 2-point statistics using phaserecovery algorithms, Acta Materialia 56 (2008) 942-948.

[218] M. Groeber, S. Ghosh, M. D. Uchic, D. M. Dimiduk, A framework for automated analysis and simulation of 3D polycrystalline microstructures.: Part 1: Statistical characterization, Acta Materialia 56 (2008) 1257 - 1273.

[219] M. Groeber, S. Ghosh, M. D. Uchic, D. M. Dimiduk, A framework for automated analysis and simulation of 3D polycrystalline microstructures. part 2: Synthetic structure generation, Acta Materialia 56 (2008) 1274 - 1287.

[220] K. Matouš, M. Lepš, J. Zeman, M. Šejnoha, Applying genetic algorithms to selected topics commonly encountered in engineering practice, Computer Methods in Applied Mechanics and Engineering 190 (2000) 1629 - 1650.

[221] D. A. Coker, S. Torquato, Extraction of morphological quantities from a digitized medium, Journal of Applied Physics 77 (1995) 6087-6099.

[222] D. A. Coker, S. Torquato, J. H. Dunsmuir, Morphology and physical properties of Fontainebleau sandstone via a tomographic analysis, Journal of Geophysical Research 101 (1996) 17-497.

[223] G. W. Milton, The Theory of Composites, volume 6, Cambridge university press, 2002.

[224] D. Talbot, J. Willis, Variational principles for inhomogeneous non-linear media, IMA Journal of Applied Mathematics 35 (1985) 39-54.

[225] P. Ponte Castañeda, Three-point bounds and other estimates for strongly nonlinear composites, Physical Review B 57 (1998) 12077-12083. 
[226] J. Simo, J. Ju, On continuum damage-elastoplasticity at finite strains, Computational Mechanics 5 (1989) 375-400.

[227] Z. P. Bažant, M. Jirásek, Nonlocal integral formulations of plasticity and damage: survey of progress, Journal of Engineering Mechanics 128 (2002) 1119-1149.

[228] A. A. Gusev, Representative volume element size for elastic composites: a numerical study, Journal of the Mechanics and Physics of Solids 45 (1997) 1449-1459.

[229] P. J. Roache, Verification and Validation in Computational Science and Engineering, Hermosa, 1998.

[230] E. Strohmaier, J. Dongarra, H. Simon, M. Meuer, Top500 supercomputer sites, Accessed February, 2016. URL: http://top500.org.

[231] J. S. Vetter, Contemporary High Performance Computing: From Petascale Toward Exascale, Chapman \& Hall/CRC, 2013.

[232] U.S. Department of Energy, Advanced simulation and computing and institutional R\&D programs, Accessed February, 2016. URL: http://nnsa.energy.gov/asc.

[233] J. C. Dietrich, M. Zijlema, J. Westerink, L. Holthuijsen, C. Dawson, R. Luettich, R. Jensen, J. Smith, G. Stelling, G. Stone, Modeling hurricane waves and storm surge using integrally-coupled, scalable computations, Coastal Engineering 58 (2011) 45-65.

[234] J. C. Dietrich, S. Tanaka, J. J. Westerink, C. Dawson, R. Luettich Jr, M. Zijlema, L. H. Holthuijsen, J. Smith, L. Westerink, H. Westerink, Performance of the unstructured-mesh, SWAN+ADCIRC model in computing hurricane waves and surge, Journal of Scientific Computing 52 (2012) 468-497.

[235] C. Davis, W. Wang, S. S. Chen, Y. Chen, K. Corbosiero, M. DeMaria, J. Dudhia, G. Holland, J. Klemp, J. Michalakes, et al., Prediction of landfalling hurricanes with the advanced hurricane WRF model, Monthly weather review 136 (2008) 1990-2005.

[236] M. F. Wehner, A. A. Mirin, P. G. Eltgroth, W. P. Dannevik, C. R. Mechoso, J. D. Farrara, J. A. Spahr, Performance of a distributed memory finite difference atmospheric general circulation model, Parallel Computing 21 (1995) $1655-1675$.

[237] J. L. Payne, B. Hassan, Massively parallel computational fluid dynamics calculations for aerodynamics and aerothermodynamics applications, in: NASA HPCCPICAS Workshop Proceedings, 1999.

[238] M. J. Wright, G. V. Candler, M. Prampolini, Data-parallel lower-upper relaxation method for the navier-stokes equations, AIAA journal 34 (1996) 1371-1377.

[239] K. Sanbonmatsu, C.-S. Tung, High performance computing in biology: Multimillion atom simulations of nanoscale systems, Journal of Structural Biology 157 (2007) 470 - 480. Advances in Molecular Dynamics Simulations.

[240] G. Zhao, J. R. Perilla, E. L. Yufenyuy, X. Meng, B. Chen, J. Ning, J. Ahn, A. M. Gronenborn, K. Schulten, C. Aiken, P. Zhang, Mature HIV-1 capsid structure by cryo-electron microscopy and all-atom molecular dynamics, Nature 497 (2013) 643-646.

[241] R. W. Strange, C. W. Yong, W. Smith, S. S. Hasnain, Molecular dynamics using atomic-resolution structure reveal structural fluctuations that may lead to polymerization of human $\mathrm{Cu}-\mathrm{Zn}$ superoxide dismutase, Proceedings of the National Academy of Sciences 104 (2007) 10040-10044.

[242] S. Plimpton, Fast parallel algorithms for short-range molecular dynamics, Journal of Computational Physics 117 (1995) $1-19$.

[243] U. Landman, Materials by numbers: Computations as tools of discovery, Proceedings of the National Academy of Sciences of the United States of America 102 (2005) 6671-6678.

[244] T. Tezduyar, S. Aliabadi, M. Behr, A. Johnson, V. Kalro, M. Litke, Flow simulation and high performance computing, Computational Mechanics 18 (2004) 397-412.

[245] C. A. Lee, S. D. Gasster, A. Plaza, C. I. Chang, B. Huang, Recent developments in high performance computing for remote sensing: A review, IEEE Journal of Selected Topics in Applied Earth Observations and Remote Sensing 4 (2011) $508-527$

[246] I. Sbalzarini, J. Walther, M. Bergdorf, S. Hieber, E. Kotsalis, P. Koumoutsakos, PPM - A highly efficient parallel particle-mesh library for the simulation of continuum systems, Journal of Computational Physics 215 (2006) 566 - 588.

[247] P. Chatelain, A. Curioni, M. Bergdorf, D. Rossinelli, W. Andreoni, P. Koumoutsakos, Billion vortex particle direct numerical simulations of aircraft wakes, Computer Methods in Applied Mechanics and Engineering 197 (2008) 1296 1304.

[248] M. Lundstrom, Moore's law forever?, Science 299 (2003) 210-211.

[249] J. Shalf, S. Dosanjh, J. Morrison, Exascale computing technology challenges, in: High Performance Computing for Computational Science-VECPAR 2010, Springer, 2011, pp. 1-25.

[250] K. Bergman, S. Borkar, D. Campbell, W. Carlson, W. Dally, M. Denneau, P. Franzon, W. Harrod, K. Hill, J. Hiller, et al., Exascale computing study: Technology challenges in achieving exascale systems, Defense Advanced Research Projects Agency Information Processing Techniques Office (DARPA IPTO), Tech. Rep 15 (2008).

[251] U.S. Department of Energy, Introducing titan: Advancing the era of accelerated computing, Accessed February, 2016. URL: https://www.olcf.ornl.gov/titan/.

[252] U.S. Department of Energy, Summit: Scale new heights. discover new solutions., 2016. URL: https://www.olcf.ornl.gov/summit/.

[253] U.S. Department of Energy, Aurora, Accessed October, 2015. URL: aurora.alcf.anl.gov.

[254] U.S. Department of Energy, Coral/sierra, Accessed February, 2016. URL: https://asc.1lnl.gov/coral-info.

[255] P. F. Fischer, K. Heisey, M. Min, Scaling limits for pde-based simulation, in: 22nd AIAA Computational Fluid Dynamics Conference, 2015, p. 3049.

[256] K. Schneider, O. V. Vasilyev, Wavelet methods in computational fluid dynamics, Annual Review of Fluid Mechanics 42 (2010) 473-503. 
[257] P. Moin, , K. Mahesh, Direct numerical simulation: A tool in turbulence research, Annual Review of Fluid Mechanics 30 (1998) 539-578.

[258] A. H. Baker, T. Gamblin, M. Schulz, U. M. Yang, Challenges of scaling algebraic multigrid across modern multicore architectures, in: Parallel \& Distributed Processing Symposium (IPDPS), 2011 IEEE International, IEEE, 2011, pp. $275-286$.

[259] T. M. Evans, G. G. Davidson, R. N. Slaybaugh, K. Clarno, Three-dimensional full core power calculations for pressurized water reactors, in: Journal of Physics: Conference Series, SciDAC, volume 68, 2010, pp. 367-379.

[260] J. Okada, T. Washio, T. Hisada, Study of efficient homogenization algorithms for nonlinear problems, Computational Mechanics 46 (2010) 247-258.

[261] F. V. Souza, D. H. Allen, Multiscale modeling of impact on heterogeneous viscoelastic solids containing evolving microcracks, International Journal for Numerical Methods in Engineering 82 (2010) 464-504.

[262] H. Talebi, M. Silani, S. P. Bordas, P. Kerfriden, T. Rabczuk, A computational library for multiscale modeling of material failure, Computational Mechanics (2013) 1-25.

[263] J. Ju, Energy-based coupled elastoplastic damage models at finite strains, Journal of Engineering Mechanics 115 (1989) $2507-2525$

[264] W. Xing and A. A. Shah and P. B. Nair, Reduced dimensional Gaussian process emulators of parametrized partial differential equations based on Isomap, Proceedings of the Royal Society of London A 471 (2015) 20140697.

[265] D. Amsallem, J. Cortial, K. Carlberg, C. Farhat, A method for interpolating on manifolds structural dynamics reducedorder models, International Journal for Numerical Methods in Engineering 80 (2009) 1241-1258.

[266] V. Triantafyllidis, W. Xing, A. Shah, P. Nair, Neural network emulation of spatio-temporal data using linear and nonlinear dimensionality reduction, in: A. H. Sulaiman, A. M. Othman, I. M. F. Othman, A. Y. Rahim, C. N. Pee (Eds.), Advanced Computer and Communication Engineering Technology: Proceedings of ICOCOE 2015, Springer, 2016, pp. 1015-1029.

[267] J. Ghaboussi, J. H. Garret Jr, X. Wu, Knowledge-based modeling of material behavior with neural networks, Journal of Engineering Mechanics 117 (1991) 132-153.

[268] S. Bhattacharjee, K. Matouš, A nonlinear manifold-based reduced order model for multiscale analysis of heterogeneous hyperelastic materials, Journal of Computational Physics 313 (2016) 635-653.

[269] D. Wirtz, N. Karajan, B. Haasdonk, Surrogate modeling of multiscale models using kernel methods, International Journal for Numerical Methods in Engineering 101 (2015) 1-28.

[270] C. Wang, S. Mahadevan, Multiscale manifold learning, in: AAAI, 2013.

[271] J. Yvonnet, Q.-C. He, The reduced model multiscale method (R3M) for the non-linear homogenization of hyperelastic media at finite strains, Journal of Computational Physics 223 (2007) 341-368.

[272] J. E. Andrade, X. Tu, Multiscale framework for behavior prediction in granular media, Mechanics of Materials 41 (2009) 652-669.

[273] G. Dvorak, Transformation field analysis of inelastic composite materials, Proc. R. Soc. Lond. A 437 (1992) $311-327$.

[274] S. Roussette, J. Michel, P. Suquet, Non uniform transformation field analysis of elastic-viscoplastic composites, Compos. Sci. Technol. 69 (2009) 22-27.

[275] J.-C. Michel, P. Suquet, A model-reduction approach to the micromechanical analysis of polycrystalline materials, Computational Mechanics (2016) 1-26.

[276] C. Oskay, J. Fish, Eigendeformation-based reduced order homogenization for failure analysis of heterogeneous materials, Computer Methods in Applied Mechanics and Engineering 196 (2007) 1216-1243.

[277] I. Jolliffe, Principal component analysis, Springer Verlag, New York, 2002.

[278] J. A. Hernández, J. Oliver, A. E. Huespe, M. A. Caicedo, J. C. Cante, High-performance model reduction techniques in computational multiscale homogenization, Computer Methods in Applied Mechanics and Engineering 276 (2014) $149-189$.

[279] P. Kerfriden, J. Rodenas, S. Bordas, Certification of projection-based reduced order modelling in computational homogenisation by the constitutive relation error, International Journal for Numerical Methods in Engineering 97 (2014) 395-422.

[280] S. Boyaval, Reduced-basis approach for homogenization beyond the periodic setting, Multiscale Modeling \& Simulation 7 (2008) 466-494.

[281] E. Monteiro, J. Yvonnet, Q. He, Computational homogenization for nonlinear conduction in heterogeneous materials using model reduction, Computational Materials Science 42 (2008) $704-712$.

[282] M. Redeker, B. Haasdonk, A POD-EIM reduced two-scale model for crystal growth, Advances in Computational Mathematics 41 (2014) 987-1013.

[283] A. Abdulle, P. Henning, A reduced basis localized orthogonal decomposition, Journal of Computational Physics 295 (2015) $379-401$.

[284] Y. Efendiev, J. Galvis, T. Y. Hou, Generalized multiscale finite element methods GMsFEM, Journal of Computational Physics 251 (2013) $116-135$.

[285] F. El Halabi, D. González, A. Chico, M. Doblaré, FE $\mathrm{FE}^{2}$ multiscale in linear elasticity based on parametrized microscale models using proper generalized decomposition, Computer Methods in Applied Mechanics and Engineering 257 (2013) 183-202.

[286] G. Box, N. Draper, Response Surfaces, Mixtures, and Ridge Analyses, Wiley Series in Probability and Statistics, Wiley, 2007.

[287] J. Yvonnet, E. Monteiro, Q. He, Computational homogenization method and reduced database model for hyperelastic heterogeneous structures, International Journal for Multiscale Computational Engineering 11 (2013) 201-225.

[288] I. Temizer, P. Wriggers, An adaptive method for homogenization in orthotropic nonlinear elasticity, Comput. Meth. 
Appl. Mech. Eng. 35-36 (2007) 3409-3423.

[289] I. Temizer, T. I. Zohdi, A numerical method for homogenization in non-linear elasticity, Computational Mechanics 40 (2007) 281-298.

[290] B. Klusemann, M. Ortiz, Acceleration of material-dominated calculations via phase-space simplicial subdivision and interpolation, Numerical Methods in Engineering 103 (2015) 256-274.

[291] T. B. Blank, S. D. Brown, A. W. Calhoun, D. J. Doren, Neural network models of potential energy surfaces, The Journal of Chemical Physics 103 (1995) 4129-4137.

[292] S. Manzhos, K. Yamashita, A model for the dissociative adsorption of $\mathrm{N}_{2} \mathrm{O}$ on $\mathrm{Cu}\left(\begin{array}{lll}1 & 0 & 0\end{array}\right)$ using a continuous potential energy surface, Surface Science 604 (2010) $555-561$.

[293] S. Manzhos, T. Carrington, Using neural networks to represent potential surfaces as sums of products, The Journal of Chemical Physics 125 (2006).

[294] D. Sidarta, J. Ghaboussi, Constitutive modeling of geomaterials from non-uniform material tests, Computers and Geotechnics 22 (1998) $53-71$.

[295] Q. Fu, Y. M. Hashash, S. Jung, J. Ghaboussi, Integration of laboratory testing and constitutive modeling of soils, Computers and Geotechnics 34 (2007) 330 - 345. Special Issue on Biologically Inspired and Other Novel Computing Techniques in Geomechanics.

[296] J. F. Unger, C. Könke, Coupling of scales in multiscale simulation using neural network, Computers \& Structures 86 (2008) 1994-2003.

[297] B. A. Le, J. Yvonnet, Q.-C. He, Computational homogenization of nonlinear elastic materials using neural networks, International Journal for Numerical Methods in Engineering 104 (2015) 1061-1084. Nme.4953.

[298] T. Furukawa, G. Yagawa, Implicit constitutive modelling for viscoplasticity using neural networks, International Journal for Numerical Methods in Engineering 43 (1998) 195-219.

[299] M. Lefik, B. Schrefler, Artificial neural network as an incremental non-linear constitutive model for a finite element code, Computer Methods in Applied Mechanics and Engineering 192 (2003) 3265 - 3283. Multiscale Computational Mechanics for Materials and Structures.

[300] R. Haj-Ali, D. A. Pecknold, J. Ghaboussi, G. Z. Voyiadjis, Simulated micromechanical models using artificial neural networks, Journal of Engineering Mechanics 127 (2001) 730-738.

[301] H. Murase, S. K. Nayar, Visual learning and recognition of 3-D objects from appearance, International journal of computer vision 14 (1995) 5-24.

[302] J. McClurkin, L. Optican, B. Richmond, T. Gawne, Concurrent processing and complexity of temporally encoded neuronal messages in visual perception, Science 253 (1991) 675-677.

[303] J. B. Tenenbaum, V. De Silva, J. C. Langford, A global geometric framework for nonlinear dimensionality reduction, Science 290 (2000) 2323-2326.

[304] V. D. Silva and J. B. Tenenbaum, Global versus local methods in nonlinear dimensionality reduction, Advances in Neural Information Processing Systems (2002) 705-712.

[305] B. Ganapathysubramanian, N. Zabaras, A non-linear dimension reduction methodology for generating data-driven stochastic input models, Journal of Computational Physics 227 (2008) 6612-6637.

[306] X. Ma, N. Zabaras, Kernel principal component analysis for stochastic input model generation, Journal of Computational Physics 230 (2011) $7311-7331$.

[307] C. g. Li, J. Guo, G. Chen, X. f. Nie, Z. Yang, A version of isomap with explicit mapping, in: Machine Learning and Cybernetics, 2006 International Conference on, 2006, pp. 3201-3206.

[308] M. Belkin, P. Niyogi, Laplacian eigenmaps for dimensionality reduction and data representation, Neural Computation 15 (2003) 1373-1396.

[309] S. T. Roweis, L. K. Saul, Nonlinear dimensionality reduction by locally linear embedding, Science 290 (2000) $2323-2326$.

[310] V. D. Maaten and JP. Laurens and E. O. Postma and H. J. van den Herik, Dimensionality reduction: A comparative review, Journal of Machine Learning Research 10 (2009) 66-71.

[311] R. R. Coifman and S. Lafon, Diffusion maps, Applied and Computational Harmonic Analysis 21 (2006) 5-30.

[312] B. Schölkopf and A. Smola and K. R. Müller, Kernel principal component analysis, Artificial Neural Networks-ICANN'97 (1997) 583-588.

[313] W. L. Oberkampf, F. G. Blottner, Issues in computational fluid dynamics code verification and validation, AIAA journal 36 (1998) 687-695.

[314] K. Salari, P. Knupp, Code verification by the method of manufactured solutions, Technical Report, Sandia National Labs., Albuquerque, NM (US); Sandia National Labs., Livermore, CA (US), 2000.

[315] M. Beneš, K. Matouš, Asynchronous multi-domain variational integrators for nonlinear hyperelastic solids, Computer Methods in Applied Mechanics and Engineering 199 (2010) 1992-2013.

[316] K. Kamojjala, R. Brannon, A. Sadeghirad, J. Guilkey, Verification tests in solid mechanics, Engineering with Computers $31(2013)$ 193-213.

[317] J. R. Kamm, J. S. Brock, S. T. Brandon, D. L. Cotrell, B. Johnson, P. Knupp, W. Rider, T. Trucano, V. G. Weirs, Enhanced Verification Test Suite for Physics Simulation Codes, Technical Report LLNL-TR-411291, Lawrence Livermore National Laboratory (LLNL), Livermore, CA, 2008.

[318] I. Babuška, J. Oden, Verification and validation in computational engineering and science: basic concepts, Computer Methods in Applied Mechanics and Engineering 193 (2004) 4057 - 4066.

[319] W. L. Oberkampf, C. J. Roy, Verification and Validation in Scientific Computing, 1st ed., Cambridge University Press, New York, NY, USA, 2010.

[320] B. Hailpern, P. Santhanam, Software debugging, testing, and verification, IBM Systems Journal 41 (2002) 4-12. 
[321] P. Roache, Fundamentals of Verification and Validation, Hermosa publ., 2009.

[322] J. Oden, S. Prudhomme, Estimation of modeling error in computational mechanics, Journal of Computational Physics $182(2002) 496-515$.

[323] J. Oden, T. I. Zohdi, Analysis and adaptive modeling of highly heterogeneous elastic structures, Computer Methods in Applied Mechanics and Engineering 148 (1997) $367-391$.

[324] J. Oden, K. S. Vemaganti, Estimation of local modeling error and goal-oriented adaptive modeling of heterogeneous materials, Journal of Computational Physics 164 (2000) $22-47$.

[325] M. Beran, J. McCoy, Mean field variations in a statistical sample of heterogeneous linearly elastic solids, International Journal of Solids and Structures 6 (1970) 1035-1054.

[326] J. E. Bishop, J. M. Emery, R. V. Field, C. R. Weinberger, D. J. Littlewood, Direct numerical simulations in solid mechanics for understanding the macroscale effects of microscale material variability, Computer Methods in Applied Mechanics and Engineering 287 (2015) 262-289.

[327] S. Ghosh, G. Weber, S. Keshavarz, Multiscale modeling of polycrystalline nickel-based superalloys accounting for subgrain microstructures, Mechanics Research Communications (2015) in press.

[328] J. Neggers, J. Hoefnagels, O. van der Sluis, M. Geers, Multi-scale experimental analysis of rate dependent metalelastomer interface mechanics, Journal of the Mechanics and Physics of Solids 80 (2015) $26-36$.

[329] H. Padilla, J. Lambros, A. Beaudoin, I. Robertson, Relating inhomogeneous deformation to local texture in zirconium through grain-scale digital image correlation strain mapping experiments, International Journal of Solids and Structures 49 (2012) $18-31$.

[330] B. M. Patterson, N. L. Cordes, K. Henderson, J. J. Williams, T. Stannard, S. S. Singh, A. R. Ovejero, X. Xiao, M. Robinson, N. Chawla, In situ x-ray synchrotron tomographic imaging during the compression of hyper-elastic polymeric materials, Journal of Materials Science 51 (2015) 171-187.

[331] W.-Y. Jang, S. Kyriakides, On the crushing of aluminum open-cell foams: Part I. Experiments, International Journal of Solids and Structures 46 (2009) $617-634$.

[332] J.-Y. Buffiere, E. Maire, J. Adrien, J.-P. Masse, E. Boller, In situ experiments with X ray tomography: an attractive tool for experimental mechanics, Experimental mechanics 50 (2010) 289-305.

[333] A. Scott, I. Sinclair, S. Spearing, A. Thionnet, A. R. Bunsell, Damage accumulation in a carbon/epoxy composite: Comparison between a multiscale model and computed tomography experimental results, Composites Part A: Applied Science and Manufacturing 43 (2012) 1514-1522.

[334] S. Roux, F. Hild, P. Viot, D. Bernard, Three-dimensional image correlation from X-ray computed tomography of solid foam, Composites Part A: Applied science and manufacturing 39 (2008) 1253-1265.

[335] M. Gates, J. Lambros, M. T. Heath, Towards high performance digital volume correlation, Experimental Mechanics 51 (2010) 491-507.

[336] J. Adrien, E. Maire, N. Gimenez, V. Sauvant-Moynot, Experimental study of the compression behaviour of syntactic foams by in situ X-ray tomography, Acta Materialia 55 (2007) 1667-1679.

[337] E. Maire, C. Bordreuil, L. Babout, J.-C. Boyer, Damage initiation and growth in metals. Comparison between modelling and tomography experiments, Journal of the Mechanics and Physics of Solids 53 (2005) 2411-2434.

[338] R. D. Crouch, S. B. Clay, C. Oskay, Experimental and computational investigation of progressive damage accumulation in CFRP composites, Composites Part B: Engineering 48 (2013) 59-67.

[339] R. Muñoz, F. Martínez-Hergueta, F. Gálvez, C. González, J. LLorca, Ballistic performance of hybrid 3D woven composites: experiments and simulations, Composite Structures 127 (2015) 141-151.

[340] W. L. Oberkampf, J. C. Helton, C. A. Joslyn, S. F. Wojtkiewicz, S. Ferson, Challenge problems: uncertainty in system response given uncertain parameters, Reliability Engineering \& System Safety 85 (2004) 11-19.

[341] P. J. Roache, Quantification of uncertainty in computational fluid dynamics, Annual Review of Fluid Mechanics 29 (1997) 123-160.

[342] H. N. Najm, Uncertainty quantification and polynomial chaos techniques in computational fluid dynamics, Annual Review of Fluid Mechanics 41 (2009) 35-52.

[343] W. L. Oberkampf, T. G. Trucano, Verification and validation in computational fluid dynamics, Progress in Aerospace Sciences 38 (2002) $209-272$ 